\title{
Naval Research Laboratory
}

Washington, DC 20375-5320

NRL/MR/8140--14-9555

\section{Automatic Identification System (AIS) Collection and Reach-back System: System Description}

ROBERT G. ROBERTS II

Gregory Gaspari

Matthew R. Webster

Command, Control, Communications, Computers, and Intelligence Branch

Space Systems Development Department

DonaLD M. MacKaY

Protasis Inc.

Alexandria, Virginia

WARREN O. MALL

Systems Engineering Associates International Inc.

Melbourne Beach, Florida

Mark E. Nelson

Comparative Innovations $L L C$

White Plains, Maryland

August 20, 2014

Approved for public release; distribution is unlimited. 


\begin{tabular}{|c|c|c|}
\hline \multicolumn{2}{|c|}{ REPORT DOCUMENTATION PAGE } & $\begin{array}{c}\text { Form Approved } \\
\text { OMB No. 0704-0188 }\end{array}$ \\
\hline \multicolumn{3}{|c|}{$\begin{array}{l}\text { Public reporting burden for this collection of information is estimated to average } 1 \text { hour per response, including the time for reviewing instructions, searching existing data sources, gathering and } \\
\text { maintaining the data needed, and completing and reviewing this collection of information. Send comments regarding this burden estimate or any other aspect of this collection of information, including } \\
\text { suggestions for reducing this burden to Department of Defense, Washington Headquarters Services, Directorate for Information Operations and Reports (0704-0188), 1215 Jefferson Davis Highway, } \\
\text { Suite 1204, Arlington, VA 22202-4302. Respondents should be aware that notwithstanding any other provision of law, no person shall be subject to any penalty for failing to comply with a collection of } \\
\text { information if it does not display a currently valid OMB control number. PLEASE DO NOT RETURN YOUR FORM TO THE ABOVE ADDRESS. }\end{array}$} \\
\hline $\begin{array}{l}\text { 1. REPORT DATE (DD-MM-YYYY) } \\
20-08-2014\end{array}$ & $\begin{array}{l}\text { 2. REPORT TYPE } \\
\text { Memorandum Report }\end{array}$ & $\begin{array}{l}\text { 3. DATES COVERED (From - To) } \\
\text { May } 2011 \text { - June } 2014\end{array}$ \\
\hline \multicolumn{2}{|l|}{ 4. TITLE AND SUBTITLE } & 5a. CONTRACT NUMBER \\
\hline \multirow{2}{*}{\multicolumn{2}{|c|}{$\begin{array}{l}\text { Automatic Identification System (AIS) Collection and Reach-back System: } \\
\text { System Description }\end{array}$}} & 5b. GRANT NUMBER \\
\hline & & 5c. PROGRAM ELEMENT NUMBER \\
\hline \multicolumn{2}{|l|}{ 6. AUTHOR(S) } & 5d. PROJECT NUMBER \\
\hline \multirow{2}{*}{\multicolumn{2}{|c|}{$\begin{array}{l}\text { Robert G. Roberts II, Gregory Gaspari, Matthew R. Webster, Donald M. MacKay, }{ }^{1} \\
\text { Warren O. Mall, }{ }^{2} \text { and Mark E. Nelson }{ }^{3}\end{array}$}} & 5e. TASK NUMBER \\
\hline & & 5f. WORK UNIT NUMBER \\
\hline \multirow{2}{*}{\multicolumn{2}{|c|}{$\begin{array}{l}\text { Naval Research Laboratory } \\
4555 \text { Overlook Avenue, SW } \\
\text { Washington, DC 20375-5320 }\end{array}$}} & $\begin{array}{l}\text { 8. PERFORMING ORGANIZATION REPORT } \\
\text { NUMBER }\end{array}$ \\
\hline & & NRL/MR/8140--14-9555 \\
\hline \multicolumn{2}{|c|}{ 9. SPONSORING / MONITORING AGENCY NAME(S) AND ADDRESS(ES) } & 10. SPONSOR / MONITOR'S ACRONYM(S) \\
\hline \multirow{2}{*}{$\begin{array}{l}\text { Military Sealift Command } \\
\text { Washington Navy Yard } \\
\text { Washington, DC 20398-5540 }\end{array}$} & \multirow{2}{*}{$\begin{array}{l}\text { U.S. Transportation Command } \\
\text { Scott Air Force Base, Illinois } 62225\end{array}$} & MSC and USTRANSCOM \\
\hline & & $\begin{array}{l}\text { 11. SPONSOR / MONITOR'S REPORT } \\
\text { NUMBER(S) }\end{array}$ \\
\hline
\end{tabular}

\section{DISTRIBUTION / AVAILABILITY STATEMENT}

Approved for public release; distribution is unlimited.

\section{SUPPLEMENTARY NOTES}

1Protasis Inc., 1219 Prince St., Alexandria, VA 22314

2Systems Engineering Associates International Inc., 440 Sandy Key, Melbourne Beach, FL 32951

${ }^{3}$ Comparative Innovations, LLC, P.O. Box 1504, White Plains, MD 20695

\section{ABSTRACT}

The Naval Research Laboratory (NRL) designed the Automatic Identification System (AIS) Collection and Reach-back System (ACRBS) to address a critical shortfall in the ability of the Military Sealift Command (MSC) to securely obtain and share its MSC ship position data along with the AIS data from all other surface contacts within AIS range (VHF) of the MSC ship. This document describes the NRL's design, assembly, and configuration of the ACRBS for the MSC. This document also provides technical information that is necessary to install ACRBS on a MSC host vessel.

\section{SUBJECT TERMS \\ Automated Tracking \\ Vessel Secure}

\begin{tabular}{|c|c|c|c|c|}
\hline \multicolumn{3}{|c|}{ 16. SECURITY CLASSIFICATION OF: } & 17. LIMITATION & 18. NUMBER \\
\hline $\begin{array}{l}\text { a. REPORT } \\
\text { Unclassified } \\
\text { Unlimited }\end{array}$ & \begin{tabular}{|c|} 
b. ABSTRACT \\
Unclassified \\
Unlimited
\end{tabular} & $\begin{array}{l}\text { c. THIS PAGE } \\
\text { Unclassified } \\
\text { Unlimited }\end{array}$ & $\begin{array}{l}\text { Unclassified } \\
\text { Unlimited }\end{array}$ & 50 \\
\hline
\end{tabular}

19a. NAME OF RESPONSIBLE PERSON Robert G. Roberts II 19b. TELEPHONE NUMBER (include area code) (202) 767-9196 x239

Standard Form 298 (Rev. 8-98) 



\section{CONTENTS}

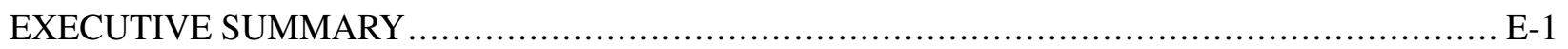

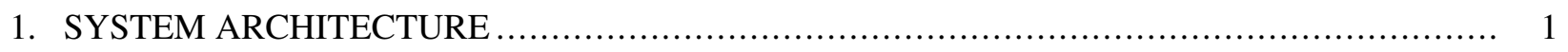

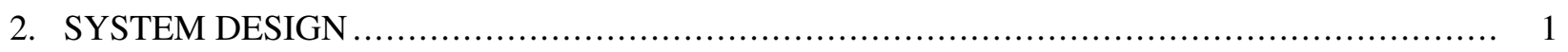

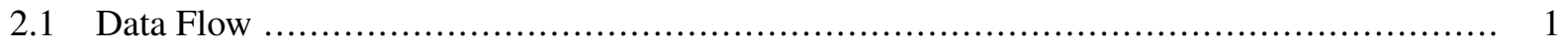

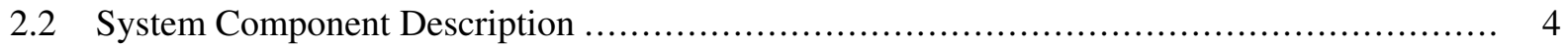

3. HOST PLATFORM SUBSYSTEM HARDWARE DESIGN $\ldots \ldots \ldots \ldots \ldots \ldots \ldots \ldots \ldots \ldots \ldots \ldots \ldots \ldots$

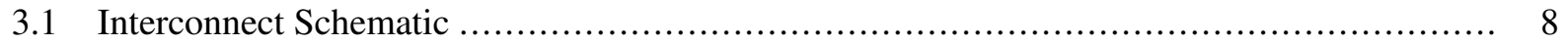

3.2 Red Processor Chassis ...................................................................... 8

3.3 Black Processor Chassis ................................................................. 18

4. SOFTWARE DESIGN........................................................................ 23

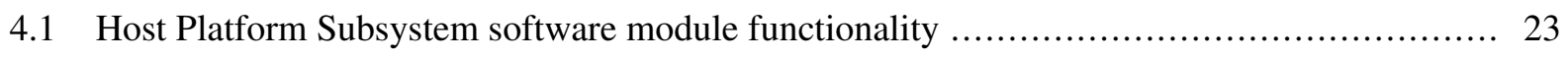

4.2 Vehicle Tracking Subsystem software module functionality .............................. 32

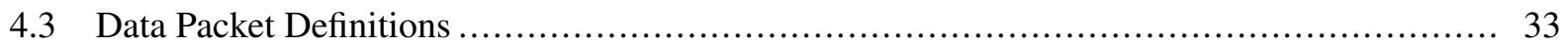

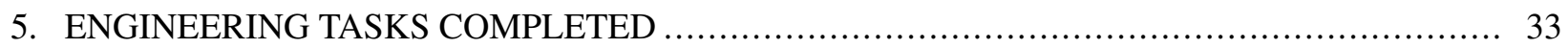

5.1 Host Platform Subsystem Hardware ..................................................... 33

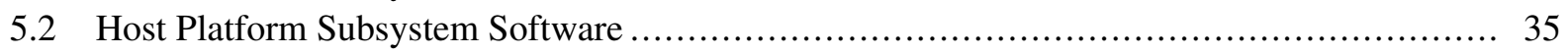

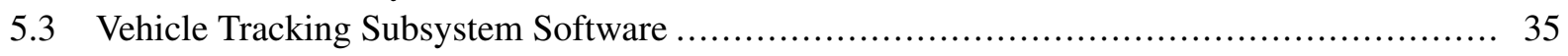

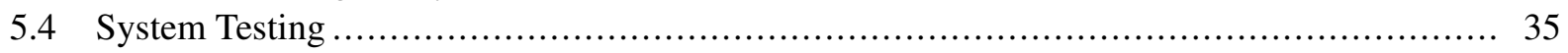

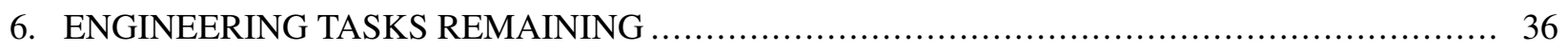

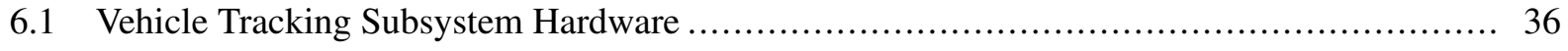

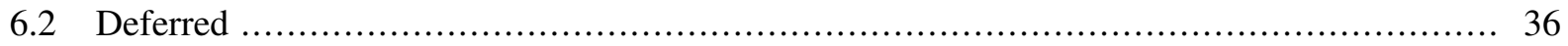

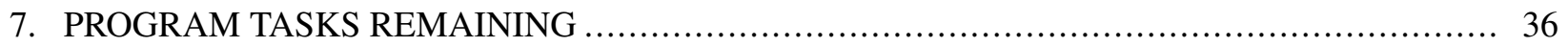

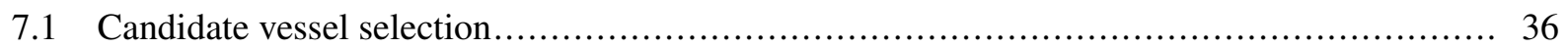

7.2 GCCS-J configuration ................................................................... 38

7.3 Documentation for Interim Authority to Test........................................ 38

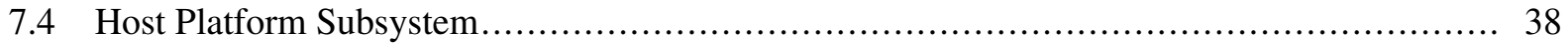

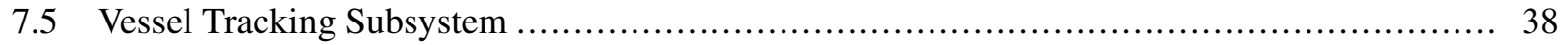

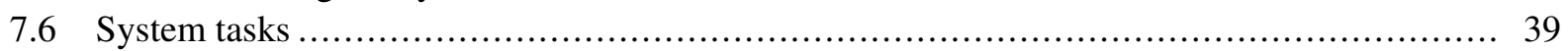

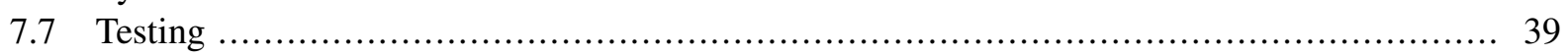

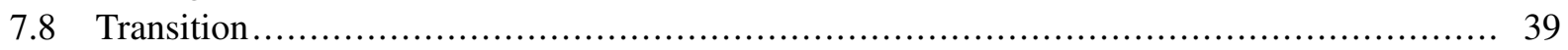

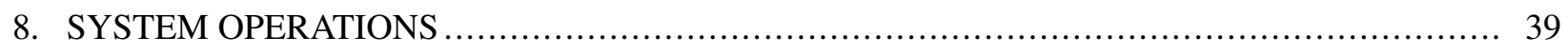

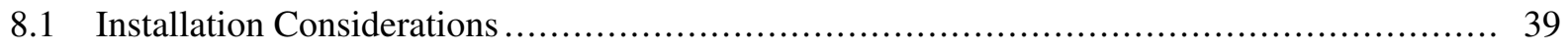




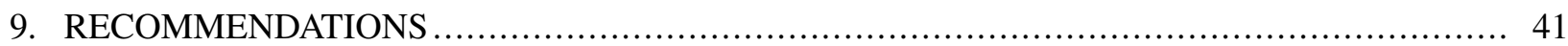

9.1 Communications Security Device, TACLANE-C100 ....................................... 41

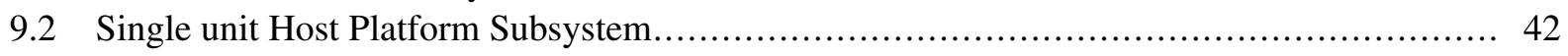

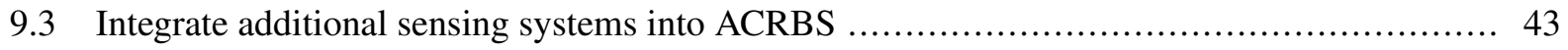

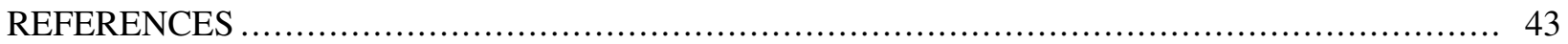

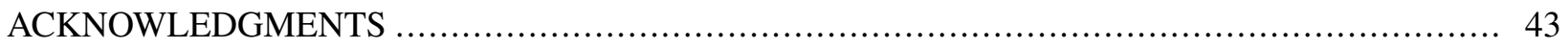

APPENDIX A-List of Acronyms......................................................................... 45 


\section{EXECUTIVE SUMMARY}

The AIS Collection and Reach-back System (ACRBS) project addresses a critical shortfall in the Military Sealift Command (MSC)'s ability to securely obtain and share MSC ship position data and Automatic Identification System (AIS) reports in the vicinity of the reporting MSC ship. The project vision was to employ ACRBS on a select set of ships as a proof-of-concept system to provide improved situational awareness that could be extended to the entire fleet.

The ACRBS unit on board the MSC ship ingests AIS data from all surface contacts within AIS range (VHF), and the Global Positioning System (GPS) position of the host MSC ship. The AIS data and GPS position are merged, and treated at the classification level of the MSC GPS report: this will be either UNCLASSIFIED, CONFIDENTIAL, or SECRET depending on the mission. The ACRBS sends the merged streams via Iridium satellite modem to a receiving ground station, where they are packetized and trans-

ported via the Defense Information System Network (DISN) to the collection point. The data is delivered in a format that can be ingested by the shore-based software application to process/display the data (e.g. Over-the-Horizon-GOLD (OTH-G) format used by GCCS-J). 


\section{AUTOMATIC IDENTIFICATION SYSTEM (AIS) COLLECTION AND REACH-BACK SYSTEM}

\section{SYSTEM DESCRIPTION}

\section{SYSTEM ARCHITECTURE}

The AIS Collection and Reach-back System (ACRBS) provides a modular systems architecture designed to combine the Global Positioning System (GPS) position of an ACRBS unit with nearby Automatic Identification System (AIS) reports, and to transmit merged reports from ACRBS units at geographically separated regions over a Wide Area Network (WAN) to a collection point. The data that an ACRBS unit collects is essentially the same as an AIS receiver, plus the GPS position of the host platform. The systems architecture combines Host Platform Subsystems (HPSs) to merge GPS-AIS reports from geographically separated regions and a Vehicle Tracking Subsystem (VTS) as the collection point for all reports.

Each HPS is self-contained and is unobtrusive to the host platform's architecture, requiring only component hardware mounting and electrical power. The HPS computes, encrypts, and transmits the host platform's positions over a Wide-area Network (WAN) to the VTS; ACRBS currently employs the Iridium Satellite Network to report the host platform's position to the VTS. Reporting intervals can be configured from seconds to weeks. The VTS receives, decrypts, and formats HPS vehicle position and contact reports for display on a Common Operational Picture (COP).

The ACRBS implementation developed specifically for the Military Sealift Command (MSC) is shown in the Operational Viewpoint (OV-1) of Fig. 1. This implementation allows for the U.S. Transportation Command (USTRANSCOM) and the MSC to maintain a near real-time tactical vehicle tracking picture consisting of high-value vehicle tracks (e.g. MSC owned and operated vessels, Ready Reserve Force (RRF) vessels, and chartered commercial vessels) and AIS contacts collected by those assets. Type 1 encryption allows for the processing of UNCLASSIFIED, CONFIDENTIAL, and SECRET position reports. The tactical asset management picture is displayed on GCCS-J.

\section{SYSTEM DESIGN}

\subsection{Data Flow}

ACRBS operates with full Type 1 encryption from the ship side to the shore-based collection platform as shown in Fig. 2. The following is an overview of the ship-to-shore data flow; see Chapter 4 below for a detailed description of the software modules and data packets.

- Ship-side data flow summary

Manuscript approved July 9, 2014. 


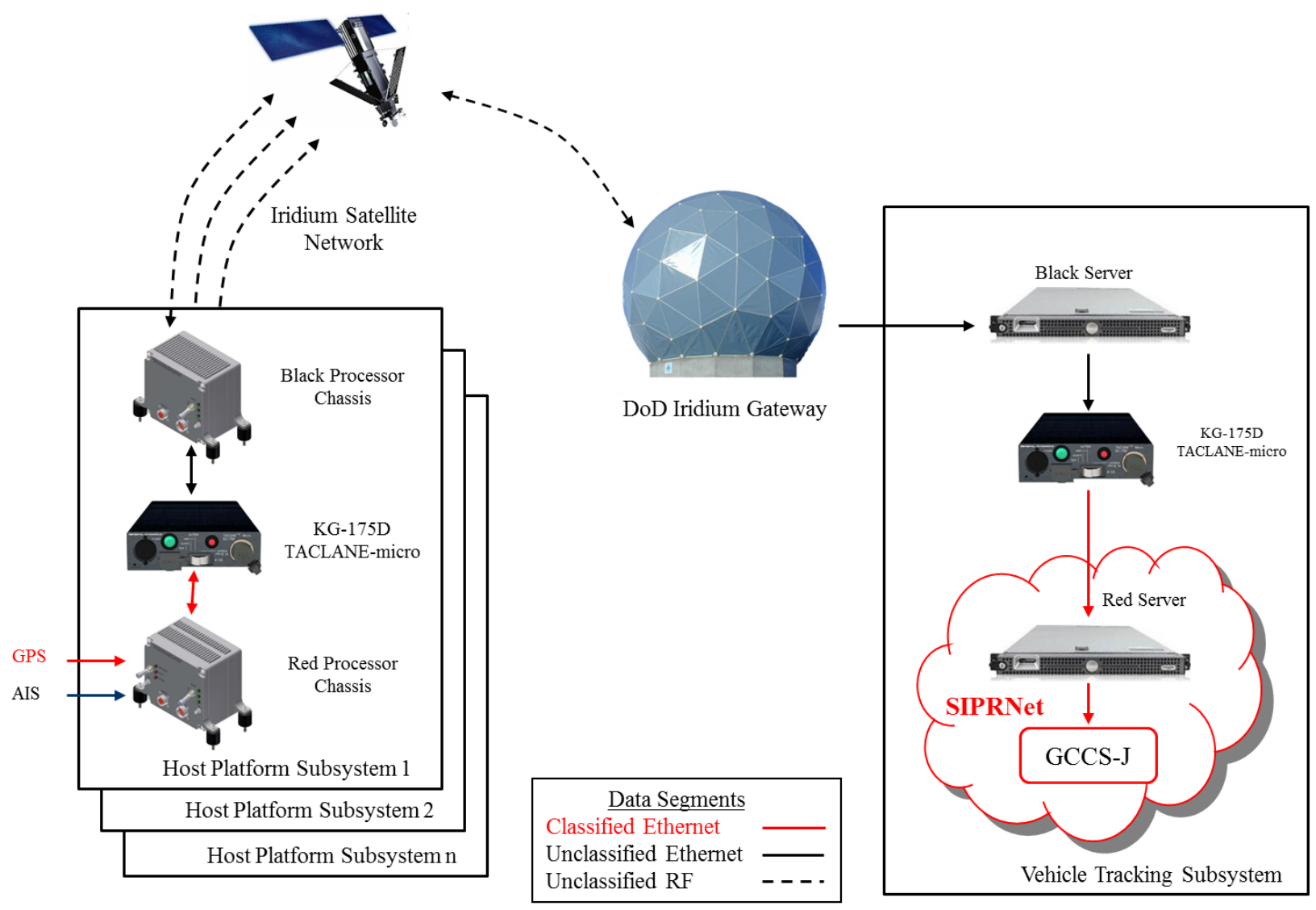

Fig. 1 - Operational Viewpoint (OV-1)

- GPS and AIS data is collected on the ship's red side and packetized into Ship Relay (ID 20) packets

- ID 20 packets are wrapped into ID 21 packets and encrypted

- encrypted ID 21 packets are sent from the ship's black side via Iridium to the shore-based collection platform

- Shore-side data flow summary

- the encrypted ID 21 packets are received by a black server at a shore site

- the encrypted ID 21 packets are decrypted and the ID 20 packets are unpacked and sent to a red server

- the ID 20 packets are processed and tracked by the SpyGlass TRK_MGR

- TRK_MGR tracks are converted to Over-the-Horizon-GOLD (OTH-G) and sent to a GCCS processor for operational display 


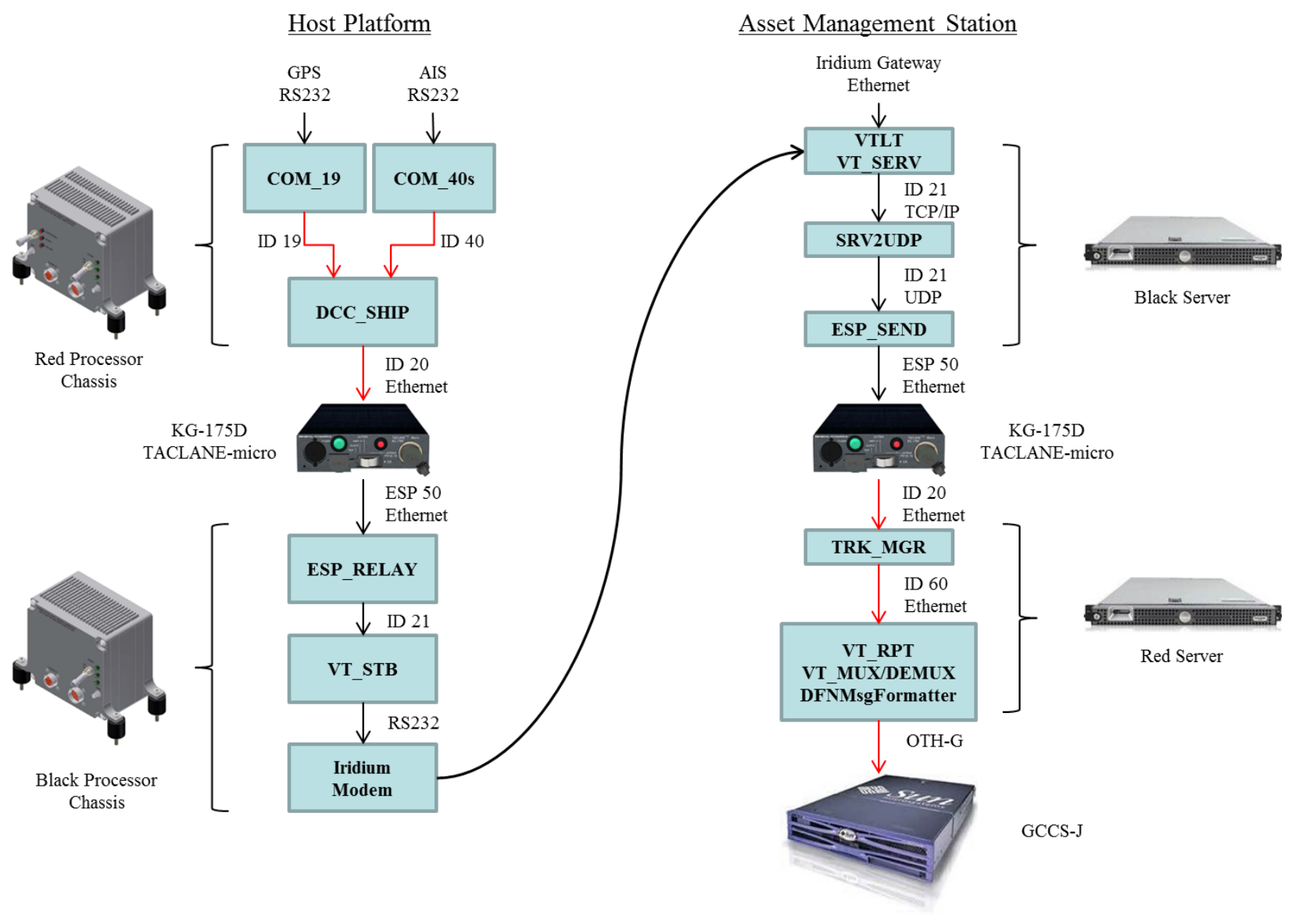

Fig. 2 - Services Viewpoint $(\mathrm{SvcV}-1)$

The ACRBS provides software modules to perform ship-side and shore-side data-and-packet processing tasks that are necessary to transfer data from the ship to the shore-based collection point. The following is an overview of the processing steps:

- Ship-side processing (see Fig. 3 below)

- COM_19 processes GPS data (raw RMC sentences) and converts it to an ID 19 packet to produce vehicle tracks

- COM_40S processes AIS data (raw AIVDM sentences) to produce ID 40 series packets

- DCC_SHIP processes Vehicle and AIS data (ID 19 and ID 40 packets) to produce Ship Relay (ID 20) packets

- ESP_RELAY and VT_SBD send the encrypted ID 21 packets over the Iridium network

- Shore-side processing (see Fig. 4 below) 
- VTL_SERV, SRV2UDP, and ESP_SEND prepare the raw ID 21 packets for decryption (blackside front-end processing)

- ESP_SEND sends the body of the ID 21 packets to the decrypter black side

- The TRK_MGR receives unencrypted ID 20 packets and establishes tracks as ID 60 series packets

- The DFN_MSG_FORMATTER converts the ID 60 series packets to OTH-G for GCCS to display

\subsection{System Component Description}

The ACRBS system components are shown in Fig. 2. The function of each component is described in the following sections.

\subsubsection{Red Processor Chassis}

The Red Processor Chassis (RPC), shown in Fig. 5, receives AIS and GPS data in NMEA 0183 format [1] using embedded receivers. The AIS and GPS data is embedded into an ID 20 binary packet, which adds a data description header that includes the host platform ID, data source, and packet checksum. This ID 21 data packet is transmitted to the HPS Communications Security (COMSEC) device via Ethernet for encrypting.

\subsubsection{Host Platform Subsystem Communications Security Device (KG-175D)}

The HPS COMSEC device, shown in Fig. 6, receives unencrypted ID 20 packets containing AIS and GPS data from the RPC and encrypts each packet using a Pre-placed key (PPK). This encryption method creates an ESP 50 packet that is transmitted to the Black Processor Chassis (BPC) via Ethernet.

Specification highlights of the KG-175D are listed below.

- Manufacturer: General Dynamics

- Model: KG-175D (TACLANE-micro)

- Certified for Top Secret/SCI and below

- Supports PPK mode which does not require negotiation with distant-end device

- Ethernet based allows multiple fielded units and a single shore-based unit

- Provides a way forward (via the C-100 device) for MSC owned or contracted vessels which do not have a Controlled Cryptographic Item (CCI) COMSEC capability

\subsubsection{Black Processor Chassis}

The BPC, shown in Fig. 5, receives the encrypted data packets (ESP 50) from the HPS COMSEC device via Ethernet. The ESP 50 data packets are embedded into an ID 21 packet that includes a data description header. This header contains the packet length, which is required by the Iridium data link. The ID 21 data packet containing which the encrypted AIS/GPS data is transmitted to the Iridium Satellite Network by the embedded Iridium modem. 

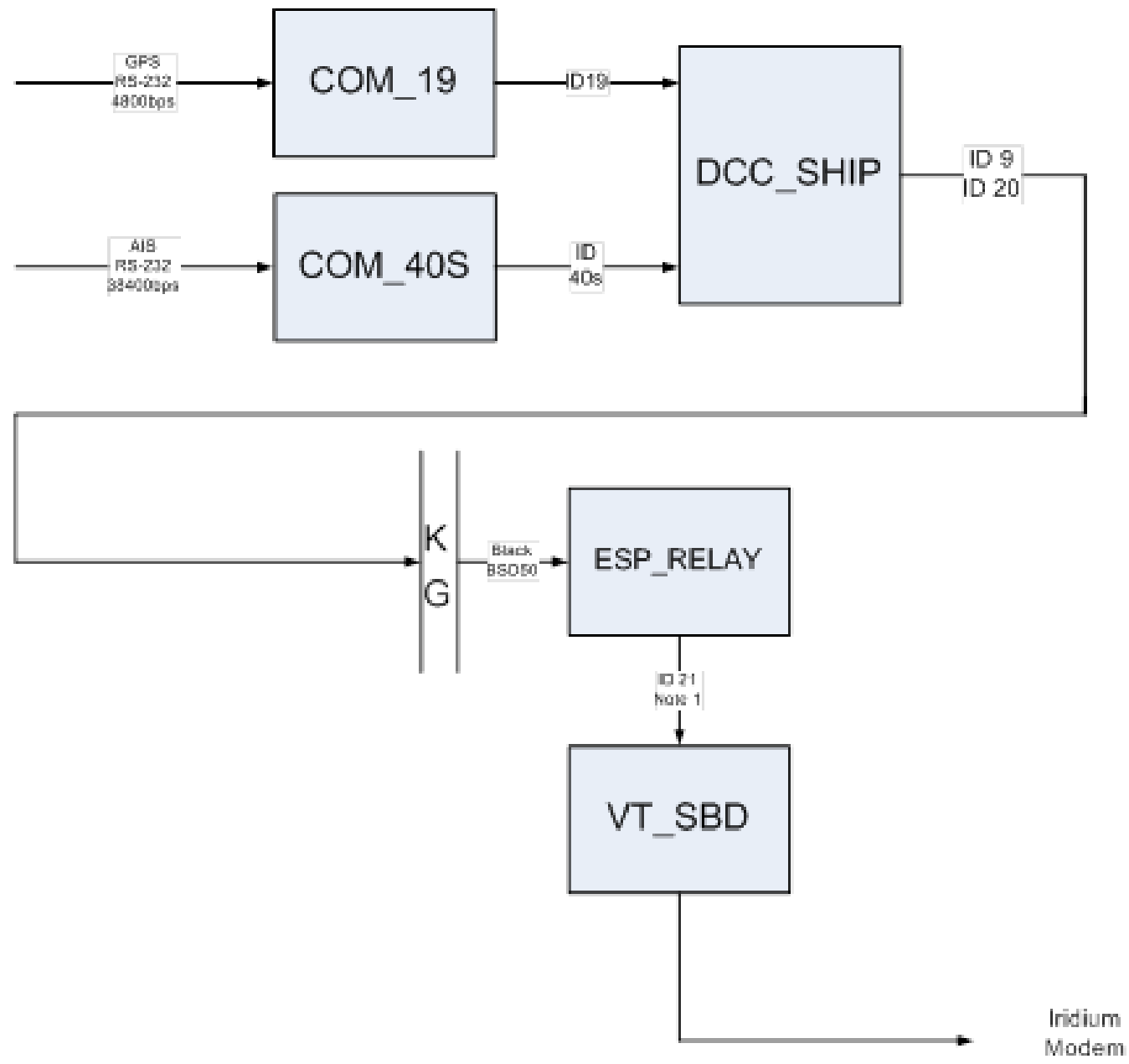

Noke1: Black BSO50

is encapsulated in

the dars field of an ID

21 pacivet

Fig. 3 - Ship-side processing 


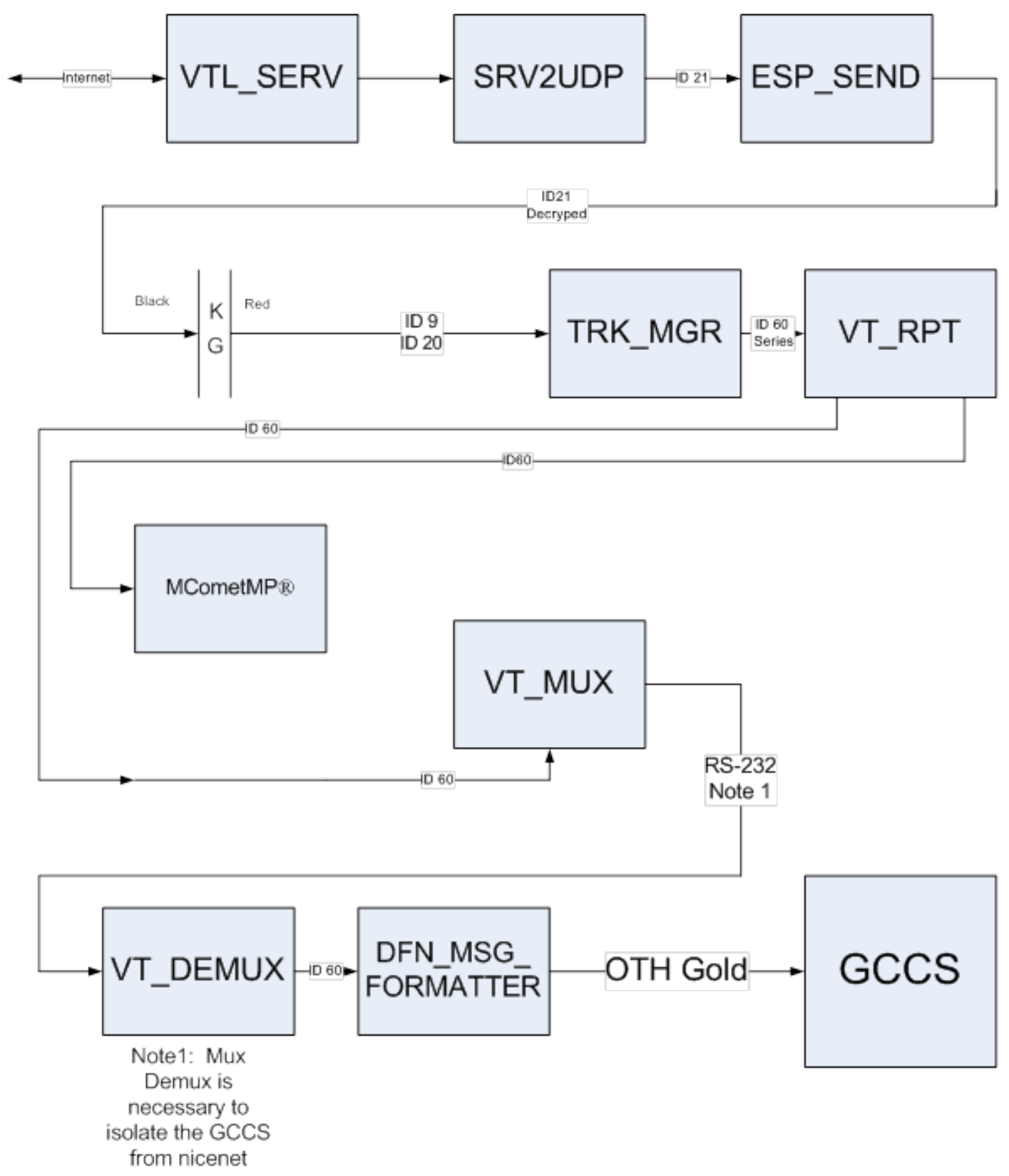

Fig. 4 - Shore-side processing 


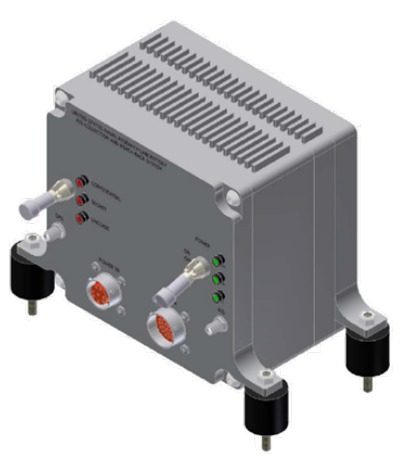

(a)

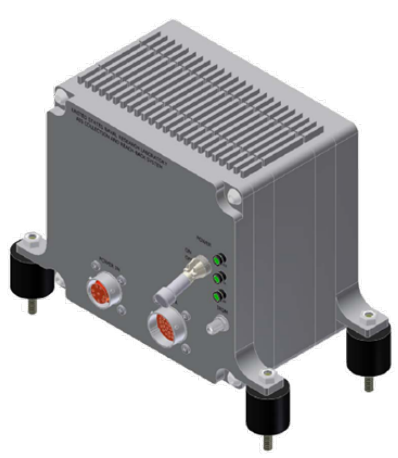

(b)

Fig. 5 - Isometric views of chassis models. (a) Red Processor Chassis. (b) Black Processor Chassis.

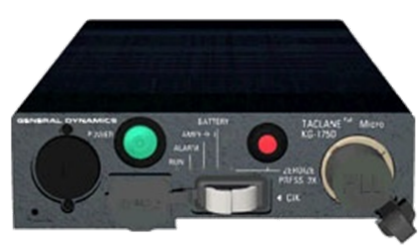

(a)

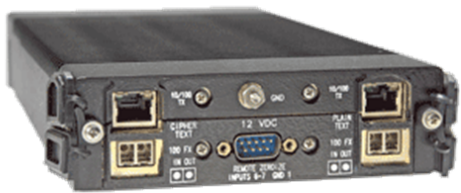

(b)

Fig. 6 - TACLANE KG-175D. (a) Front. (b) Rear.

\subsubsection{Black Server}

The Black Server provides the server function required by the Department of Defense (DoD) Iridium Gateway for delivery of Short Burst Data (SBD) packets. The Black Server receives SBD messages containing ID 21 data packets from the DoD Iridium Gateway, extracts encrypted AIS and GPS data packets (ESP 50) from the ID 21 packet, and transmits it to VTS COMSEC device via Ethernet for decrypting.

\subsubsection{Vehicle Tracking Subsystem Communications Security Device (KG-175D)}

The VTS COMSEC device receives ESP 50 data packets from the Black Server, and decrypts each packet using a PPK, which results in an ID 20 data packet consisting of header information (host platform ID, data source, and packet checksum) and embedded AIS/GPS data in NMEA 0183 format. The decrypted ID 20 data packet is transmitted to the Red Server via Ethernet.

\subsubsection{Red Server}

The Red Server receives decrypted ID 20 data packets from the VTS COMSEC device via Ethernet, converts the ID 20 track data to the OTH-G message format, and transmits the OTH-G message to the GCCS-J via Ethernet for display. 


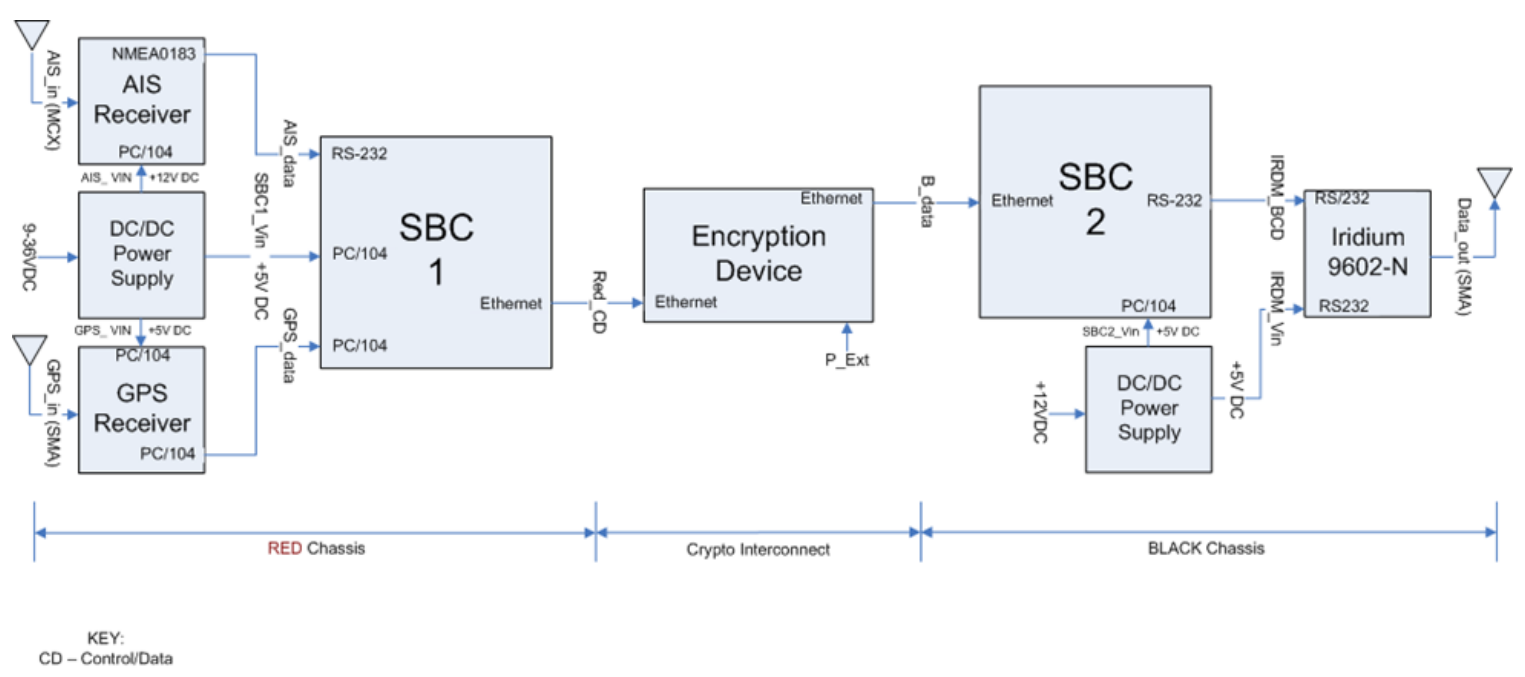

Fig. 7 - Interconnect Schematic, Top Level, Host Platform Subsystem

\section{HOST PLATFORM SUBSYSTEM HARDWARE DESIGN}

This section details the electrical and mechanical designs of the HPS.

\subsection{Interconnect Schematic}

The HPS top level interconnect schematic is shown in Fig. 7, and the detailed interconnect schematic is shown in Fig. 8.

\subsection{Red Processor Chassis}

\subsubsection{Enclosure}

The RPC enclosure provides housing for PC/104 compatible modules with a water-tight seal, shock and vibration isolators, and conduction cooling provisions. The enclosure is assembled using parts from VPI Embedded's PC/104 Rugged Enclosure System. Fig. 9 shows a sample modular slice from the manufacturer.

The RPC enclosure is constructed using a front end cap, two $1 \mathrm{U}$ modules, a $3 \mathrm{U}$ module, and an end cap as shown in Fig. 10. Each part is made from nickel-plated aluminum to provide corrosion resistance in a salt-fog environment.

The RPC front end cap is customized with mounting holes for the following components: power and data connectors; power and data classification toggle switches; power good, AIS data, and data classification indicators (LEDs); and, AIS and GPS antenna connectors. The toggle switches are of the locking type to prevent accidental position changes. The RPC front panel design layout is shown in Fig. 11.

The intent of the classification switch is to give the user the ability to set the system's data classification labels to match the classification of the host platform's position. It is the user's responsibility to set the classification switch in the appropriate position to avoid mislabeling data. The data classification feature has been implemented in hardware, but not in software. Software implementation was deferred to a future build. 


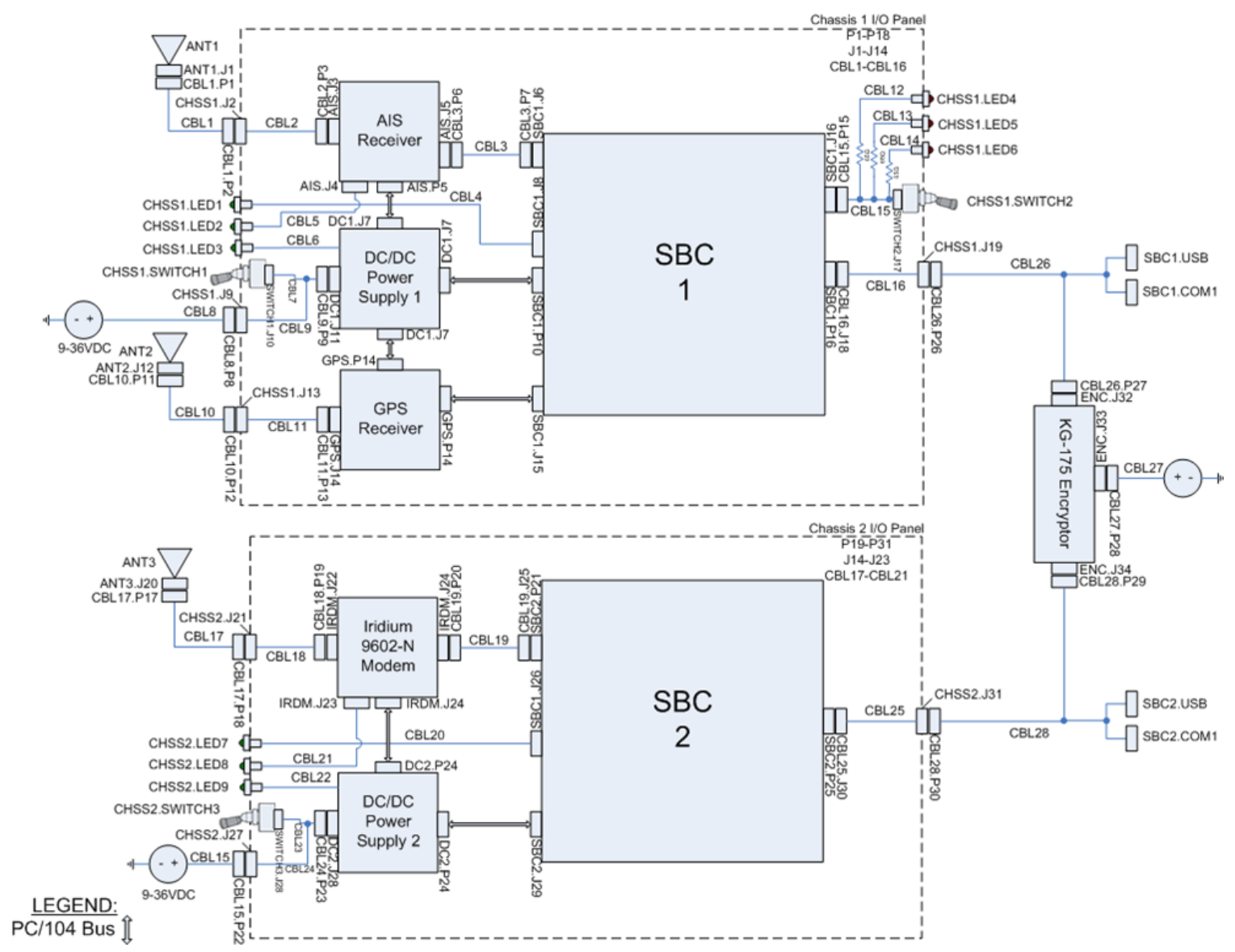

Fig. 8 - Interconnect Schematic, Detailed, Host Platform Subsystem

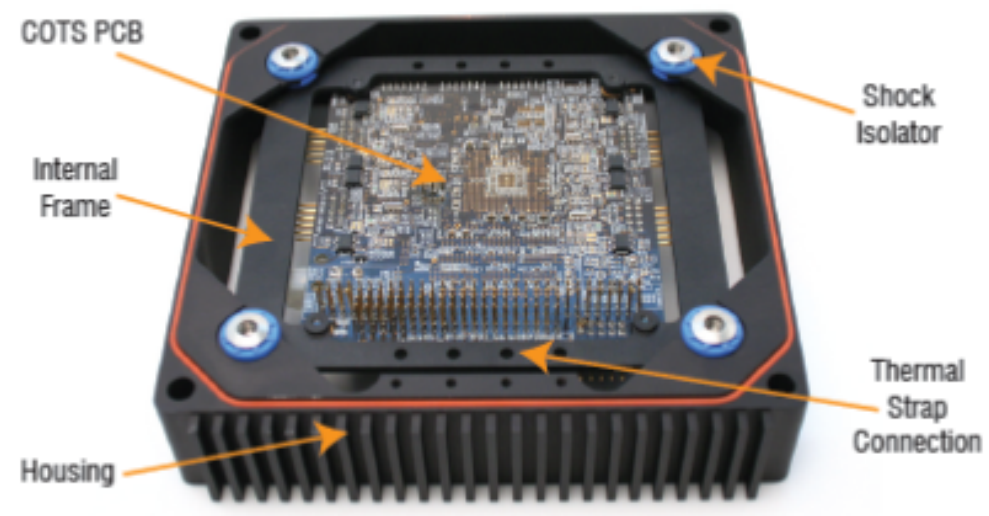

Fig. 9 - Sample modular slice from VPI Embedded's PC/104 Rugged Enclosure System 


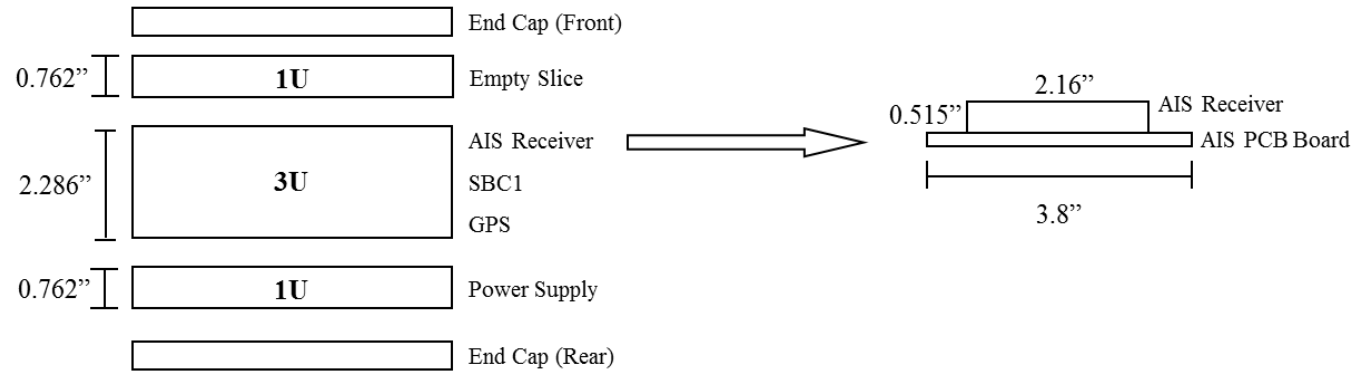

Fig. 10 - Enclosure modular slice configuration, Red Processor Chassis

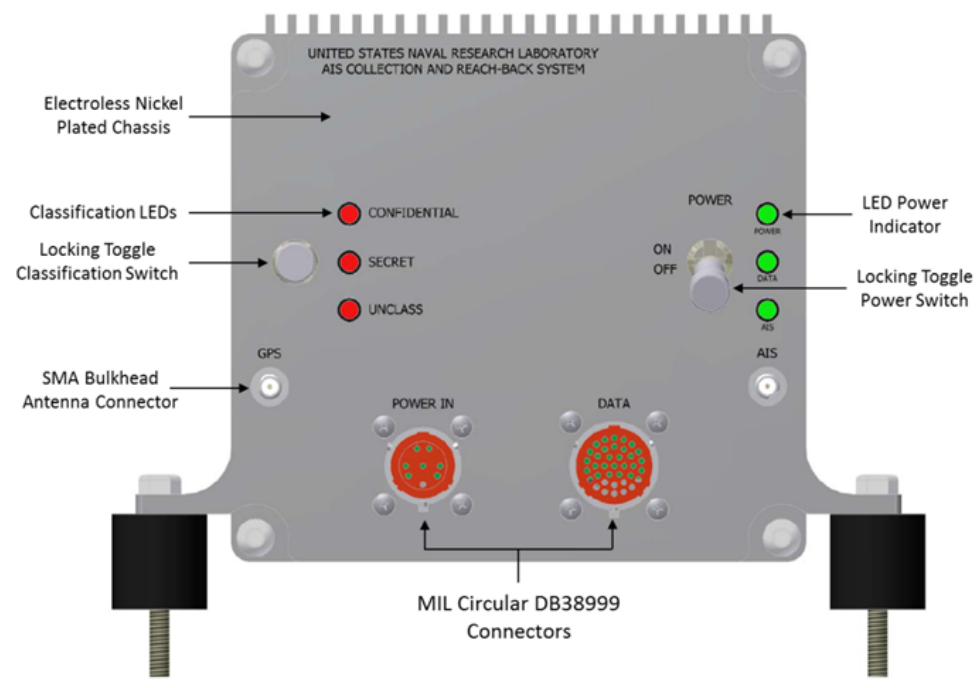

Fig. 11 - Front Panel Layout, Red Processor Chassis 


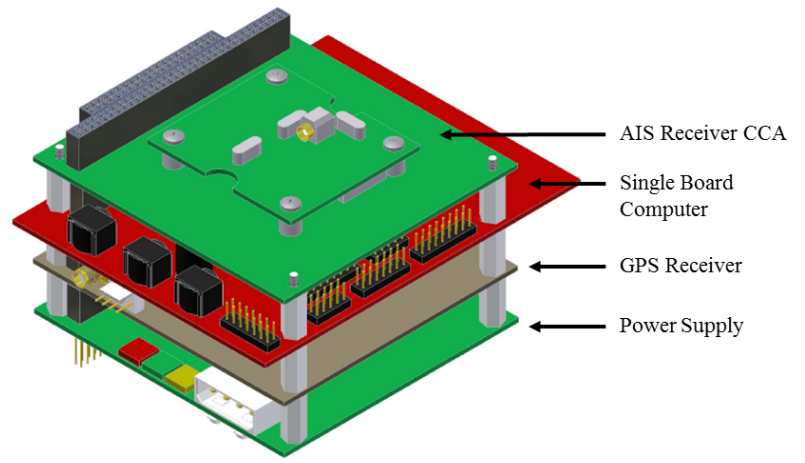

(a)

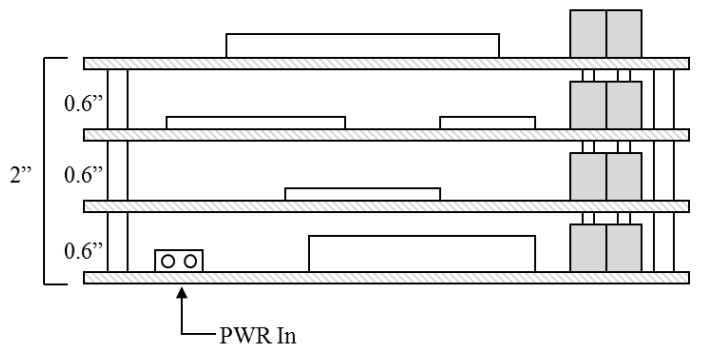

(b)

Fig. 12 - Circuit Card Stack, Red Processor Chassis. (a) 3D model. (b) Side view.

\subsubsection{Card Stack-up}

The RPC's arrangement of circuit card assemblies is shown in Fig. 12.

\subsubsection{Components}

\subsubsection{Single Board Computer}

The Single Board Computer (SBC) is shown in Fig. 13; specification highlights are listed below. The RPC and BPC use the same model SBC.

- Manufacturer: Technologic Systems Inc.

- Model: TS-7260

- 200 MHz ARM9 CPU, Fanless

- Power Requirement: $<1 \mathrm{~W}$

- 64 MB RAM, 128 MB Flash Memory

- Support for Linux 2.6

- Temperature Sensor

- Real Time Clock

- Operating Temperature: $-40^{\circ} \mathrm{C}$ to $85^{\circ} \mathrm{C}$

- 3 x COM ports

- 1 x 8-bit PC/104 Bus

- 2 x USB 2.0

- 1 x 10/100 Ethernet

- 30 x Data I/O Lines 


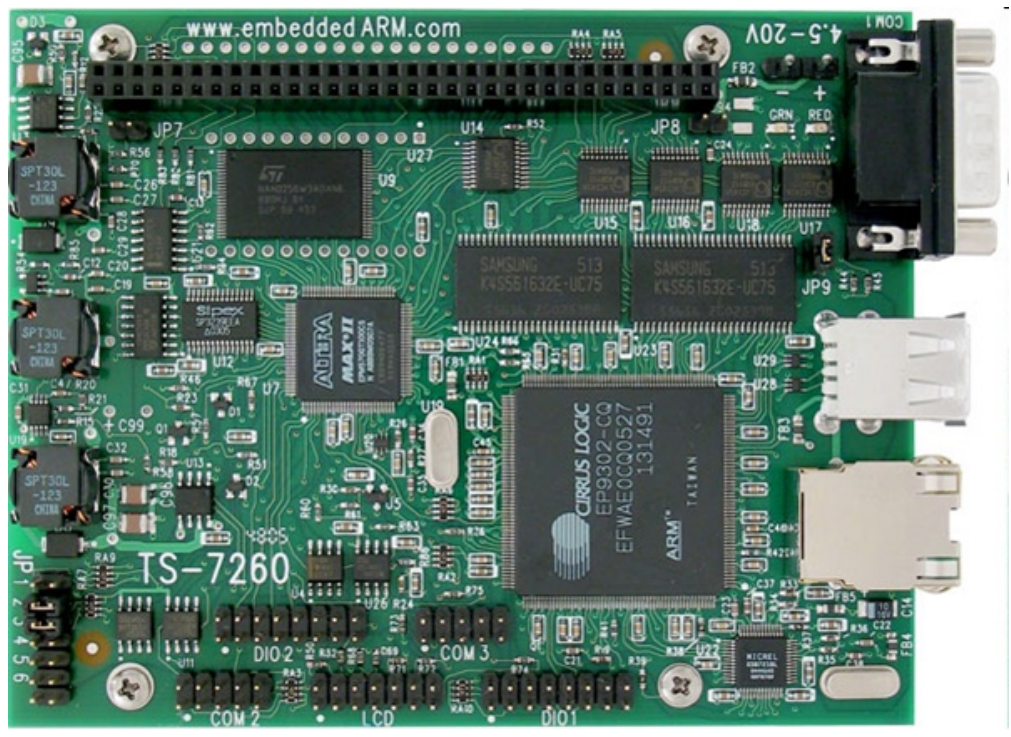

Fig. 13 - Technologic Systems TS-7260 SBC

\subsubsection{Power Supply Module}

The Power Supply Module (PSM) is shown in Fig. 14; specification highlights are listed below. The RPC and BPC use the same model PSM.

- Manufacturer: WinSystems Inc.

- Model: PCM-DC-AT512-P

- Output Voltages: 5 VDC @ 20 A; +12 VDC @ 3 A; -12 VDC @ 800 mA

- Input Voltage: 10-50 VDC

- Operating Temperature: $-40^{\circ} \mathrm{C}$ to $85^{\circ} \mathrm{C}$

- No fan or heat sink required

- No minimum load required for regulation

- Outputs with short circuit, overload and overvoltage protection

- Power-on LEDs provide visual status of power

- Screw terminals for accessory power connection

- High efficiency design

- Fast transient response

- 1 x 16-bit PC/104 Bus 


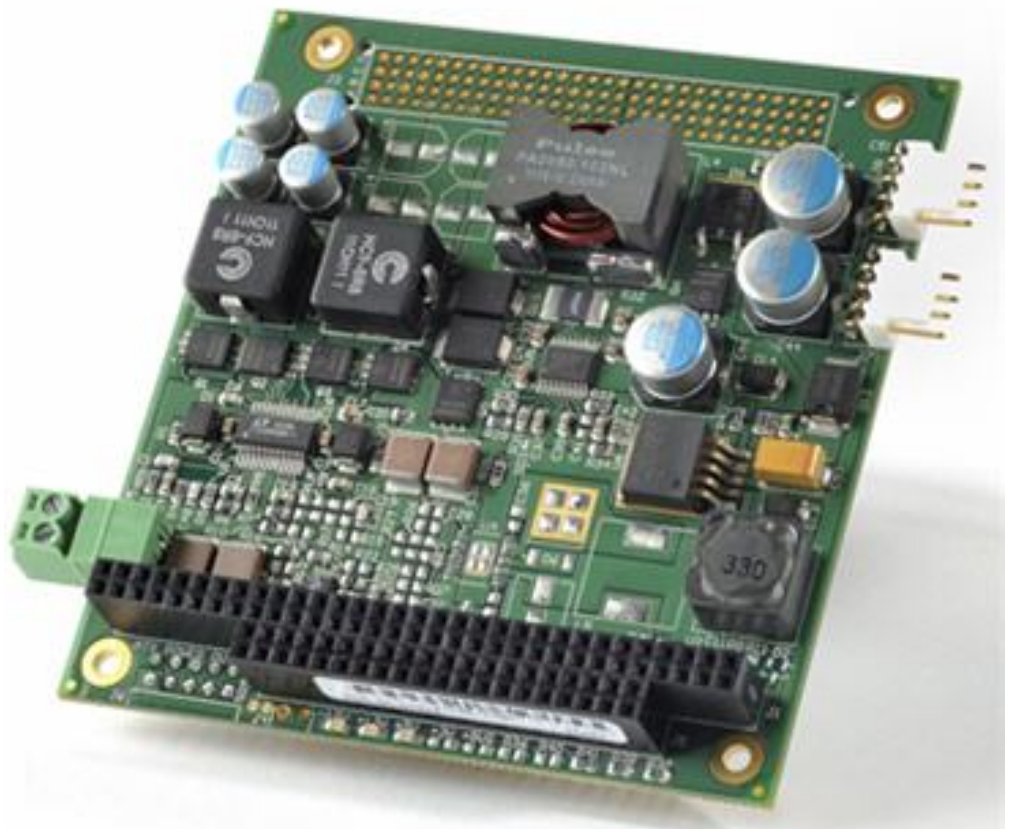

Fig. 14 - WinSystems PCM-DC/DC PSM

\subsubsection{GPS Receiver Module}

The GPS receiver module is shown in Fig. 15; specification highlights are listed below.

- Manufacturer: WinSystems Inc.

- Model: PCM-GPS

- 12-Channel Trimble Lassen iQ GPS module

- GSM/GPRS and CDMA Support

- NMEA0183, TSIP, TAIP Support

- Accuracy: $8 \mathrm{~m}$ (horizontal), $0.06 \mathrm{~m} / \mathrm{sec}$ (velocity)

- Power Req.: $50 \mathrm{~mA} @ 5 \mathrm{~V}(0.25 \mathrm{~W})$ typical

- Operating Temperature: $-40^{\circ} \mathrm{C}$ to $85^{\circ} \mathrm{C}$

- 1 x 16-bit PC/104 Bus

- 1 x SMA Antenna connector

\subsubsection{AIS Receiver Circuit Card Assembly}

The AIS Circuit Card Assembly (CCA), shown in Fig. 16, consists of a Commercial Off-The-Shelf (COTS) AIS receiver module mounted on a custom Printed Circuit Board (PCB) designed by the Naval Research Laboratory (NRL). 


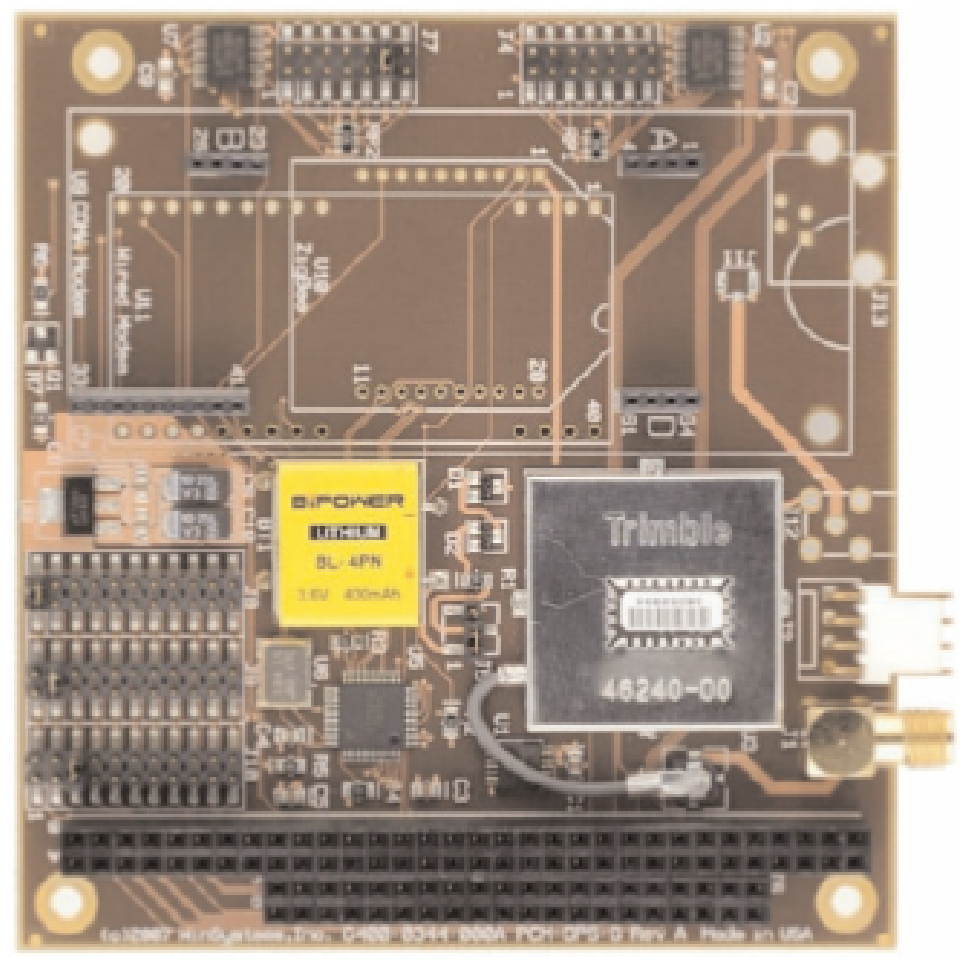

Fig. 15 - WinSystems PCM-GPS Module

The custom PCB provides PC/104 electrical and mechanical interfacing for the AIS receiver, which is not in the PC/104 form factor. Electrical interfaces include power monitoring circuitry with a power good output signal, an Ethernet port with impedance matching circuitry, a SBC interface, and a classification switch interface.

The AIS receiver module is shown in Fig. 17; specification highlights are listed below.

- Manufacturer: SRT Marine Technology

- Model: Krypton AIS Receiver PCA

- Dual Channel receiver (161.975 MHz and 162.025 MHz)

- Rx Sensitivity: <-107 dBm at $20 \%$ error rate

- Operating Temperature: $-25^{\circ} \mathrm{C}$ to $55^{\circ} \mathrm{C}$

- 1 x NMEA 0183 Output port

- 1 x USB virtual COM port

- 1 x VHF Antenna connector (MCX) 


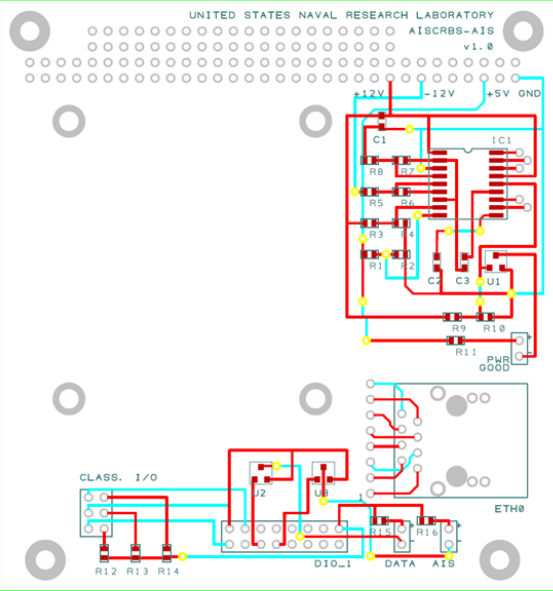

(a)
AIS Receiver Module

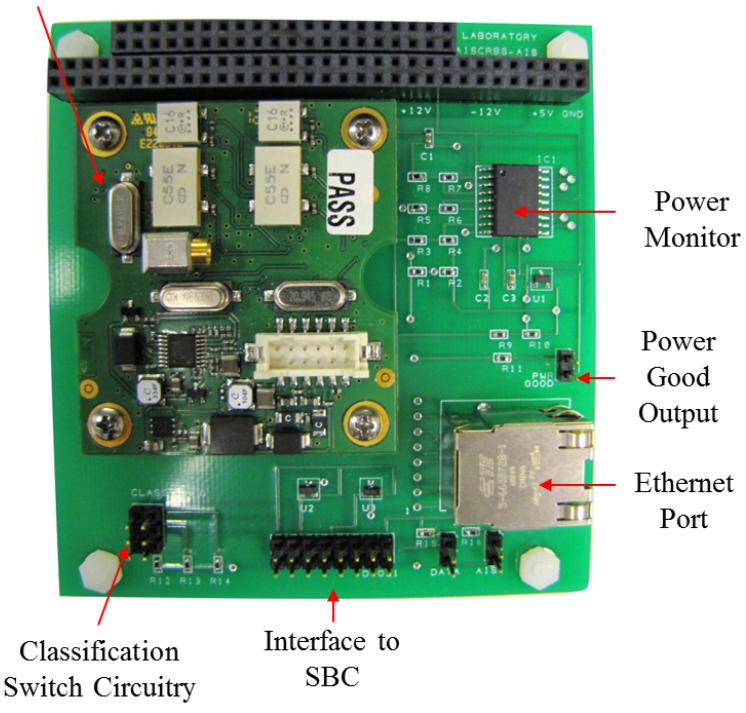

(b)

Fig. 16 - AIS Receiver Circuit Card Assembly. (a) Printed Circuit Board layout. (b) Circuit Card Assembly.

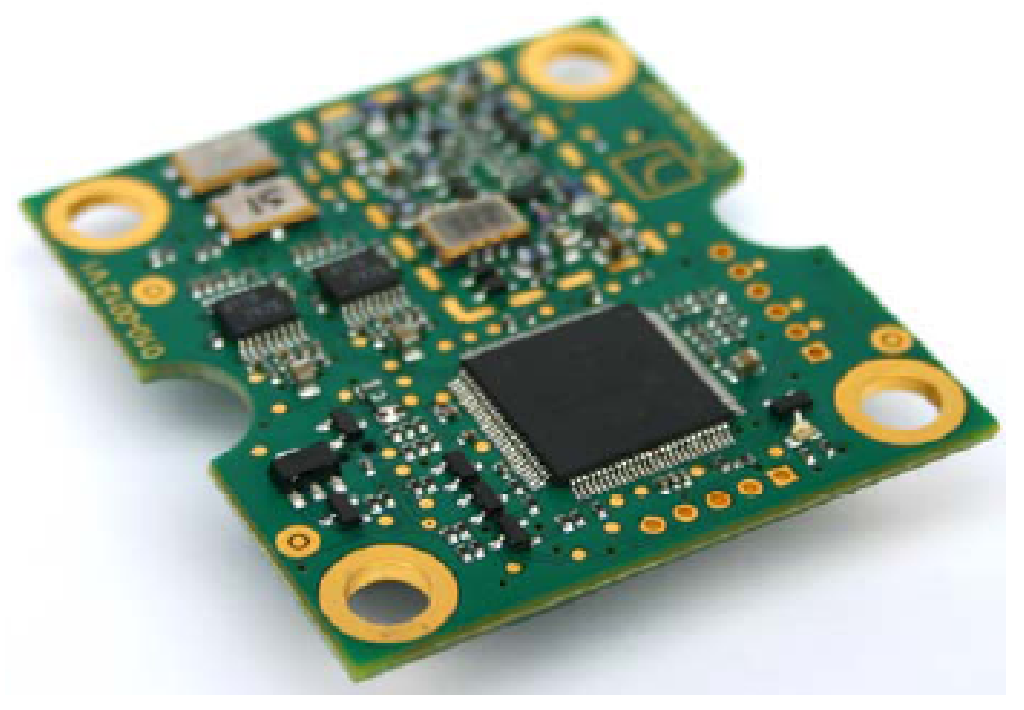

Fig. 17 - SRT Marine Technology Krypton AIS Receiver PCA 


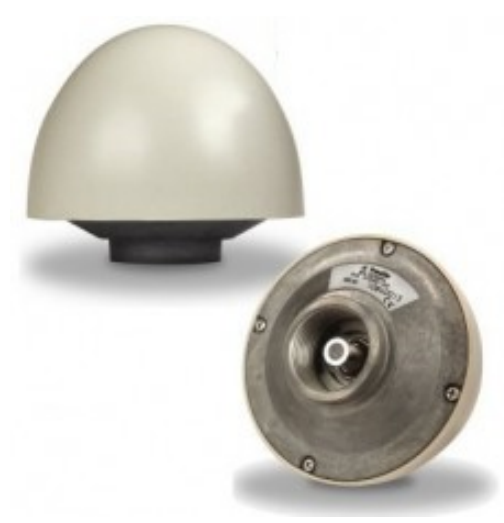

Fig. 18 - Trimble Bullet III GPS Antenna

\subsubsection{GPS Antenna}

The GPS antenna is shown in Fig. 18; specification highlights are listed below.

- Manufacturer: Trimble Navigation Limited

- Model: Bullet III GPS

- Active 3.3 VDC operation

- $30 \mathrm{~dB}$ Gain

- 100 W Power Rating

- Operating Temperature: $-40^{\circ} \mathrm{C}$ to $85^{\circ} \mathrm{C}$

- 5\% Salt Spray Tested, 96 hours

\subsubsection{AIS Antenna}

The AIS antenna is shown in Fig. 19; specification highlights are listed below.

- Manufacturer: Morad Electroncis Corp.

- Model: HD159 VHF

- Omni-Directional operation

- 156-161 MHz frequency range

- $6 \mathrm{dBi}$ Gain

- 100 W Power Rating 


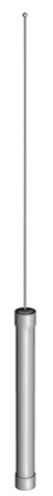

Fig. 19 - Morad HD159 VHF Antenna

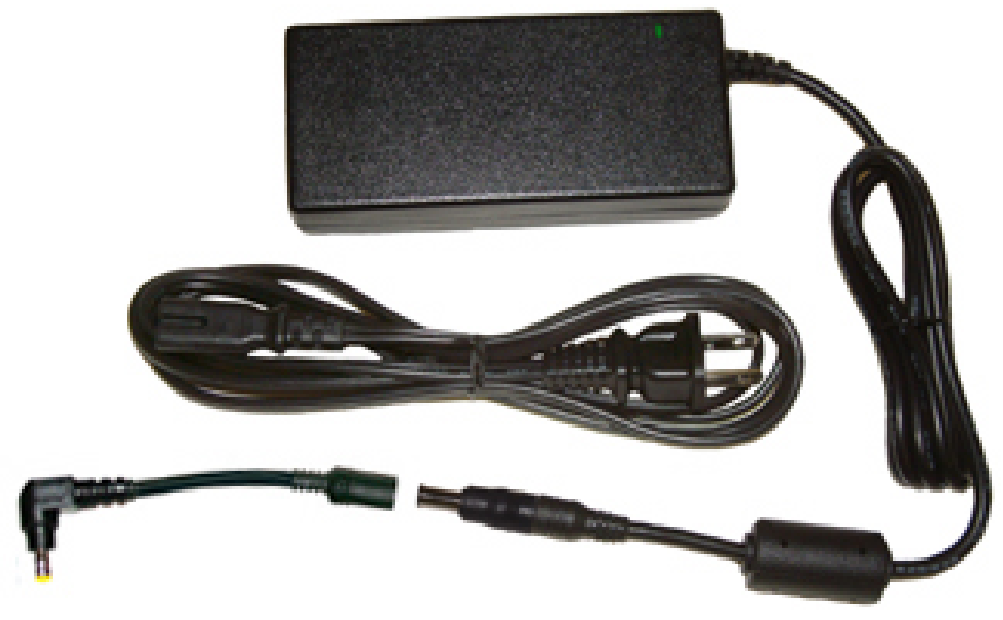

Fig. 20 - LIND AC90-PA Power Adapter

\subsubsection{AC Power Adapter}

The AC power adapter used to power the RPC and BPC is shown in Fig. 20; specification highlights are listed below.

- Manufacturer: Lind Electronics Inc.

- Model: AC90-PA

- Output Voltage: 15.6 VDC

- Output Power: $90 \mathrm{~W}$

- Output Current (max) : 4.75 A 
- Input Voltage: 100-240 VAC

- Internal resetting fuse

- Ferrite Bead for additional EMI Filtering

\subsubsection{Wiring Diagram}

The RPC wiring diagram is shown in Fig. 21.

\subsection{Black Processor Chassis}

\subsubsection{Enclosure}

The BPC enclosure provides housing for PC/104 compatible modules with a water-tight seal, shock and vibration isolators, and conduction cooling provisions. The enclosure is assembled using parts from VPI Embedded's PC/104 Rugged Enclosure System. Fig. 9 shows a sample modular slice from the manufacturer.

The BPC enclosure is constructed using a front end cap, two $1 \mathrm{U}$ modules, a $2 \mathrm{U}$ module, and an end cap as shown in Fig. 22. Each part is made from nickel-plated aluminum to provide corrosion resistance in a salt-fog environment.

The BPC front end cap is customized with mounting holes for the following components: power and data connectors; power toggle switch; power good, data, and Iridium indicators (LEDs); and, Iridium antenna connector. The toggle switch is of the locking type to prevent accidental position changes. The BPC front panel design layout is shown in Fig. 23.

\subsubsection{Card Stack-up}

The BPC's arrangement of circuit card assemblies is shown in Fig. 24.

\subsubsection{Components}

\subsubsection{Single Board Computer}

The BPC uses the same model SBC as the RPC; it is described in Section 3.2.3.1.

\subsubsection{Power Supply Module}

The BPC uses the same model PSM as the RPC; it is described in Section 3.2.3.2. 


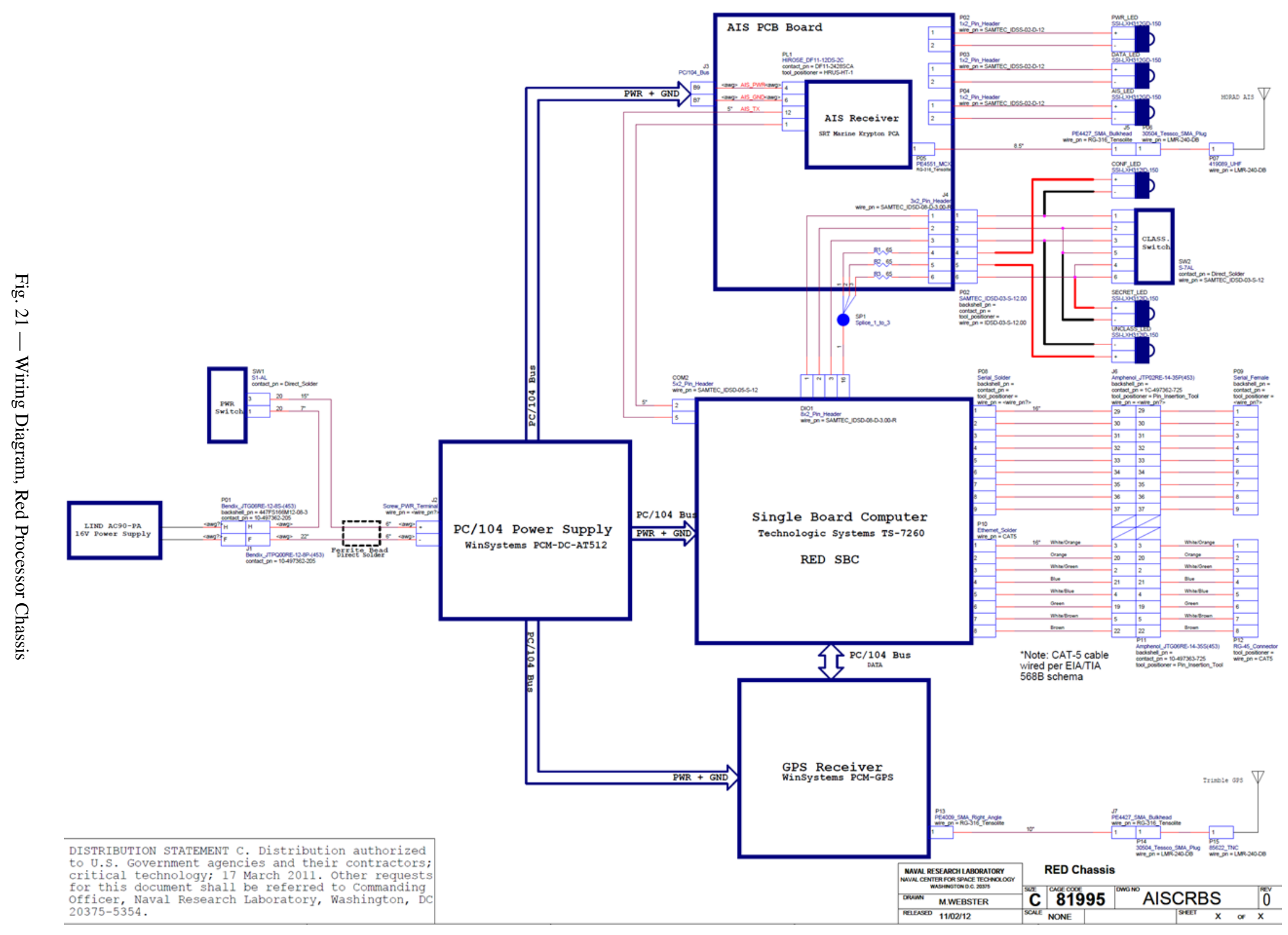




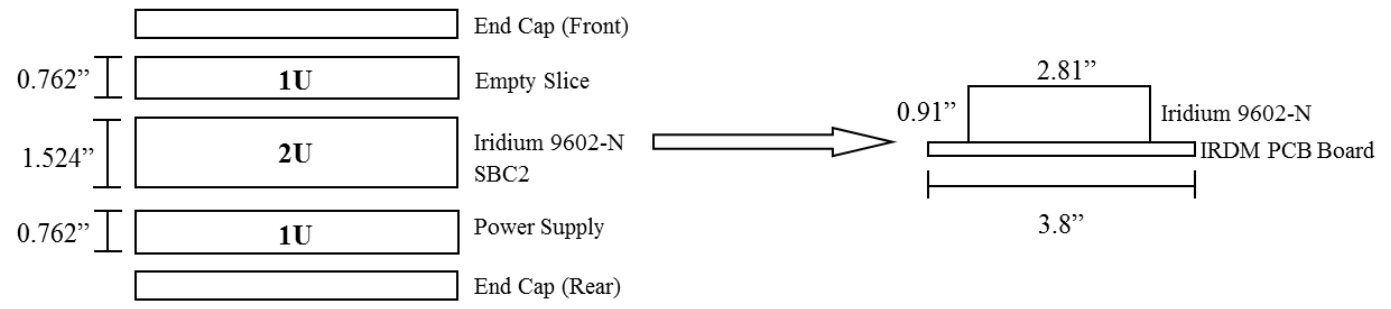

Fig. 22 - Enclosure modular slice configuration, Black Processor Chassis

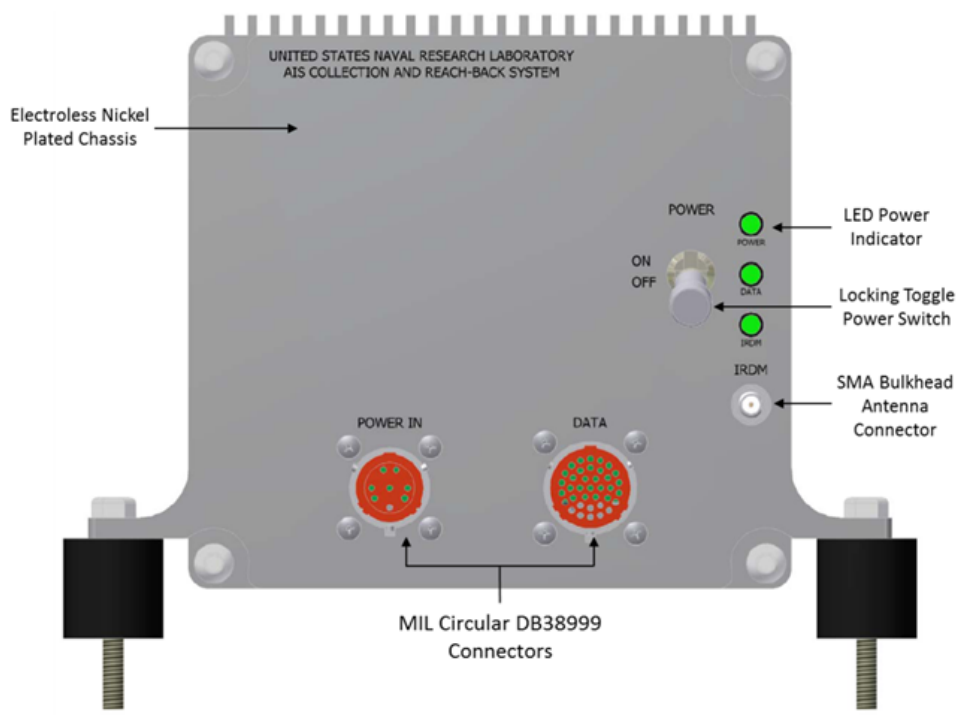

Fig. 23 - Front Panel Layout, Black Processor Chassis

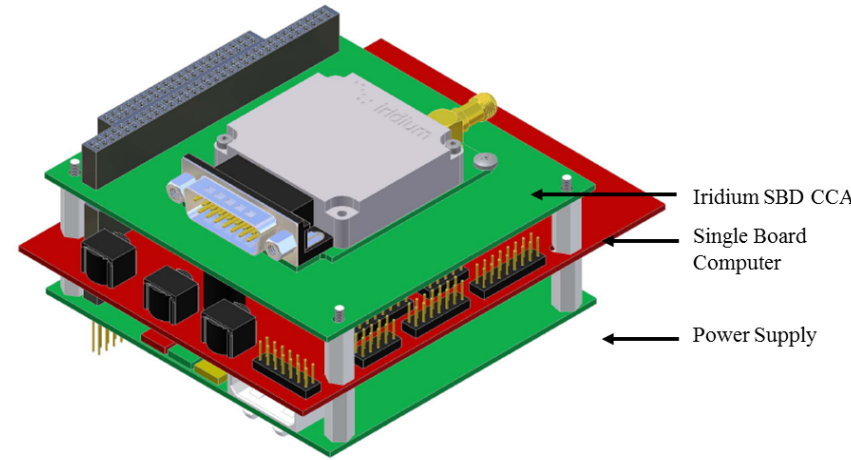

(a)

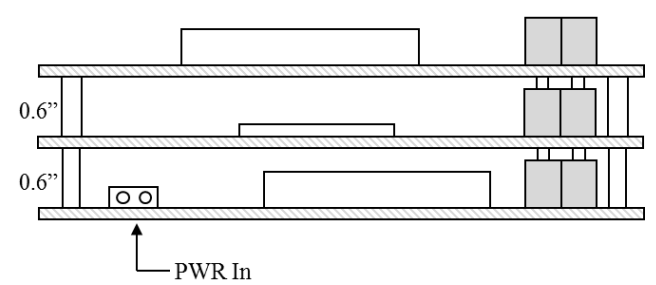

(b)

Fig. 24 - Circuit Card Stack, Black Processor Chassis. (a) 3D model. (b) Side view. 


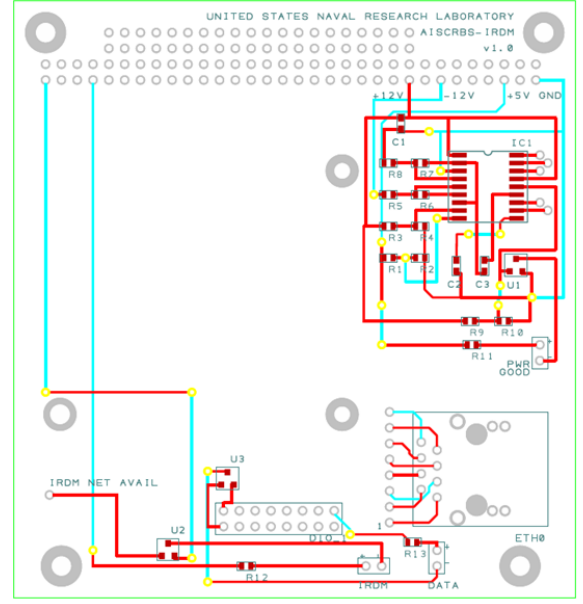

(a)

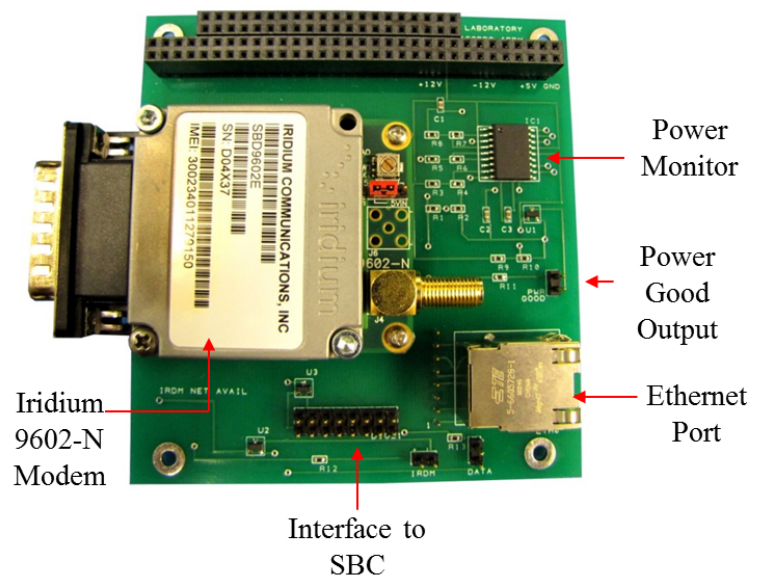

(b)

Fig. 25 - Iridium Short Burst Data Modem Circuit Card Assembly. (a) Printed Circuit Board layout.

(b) Circuit Card Assembly.

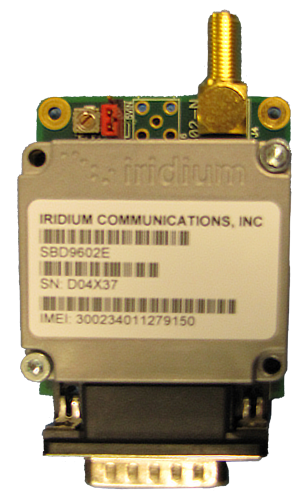

Fig. 26 - NAL Research SBD9602E short burst data modem 


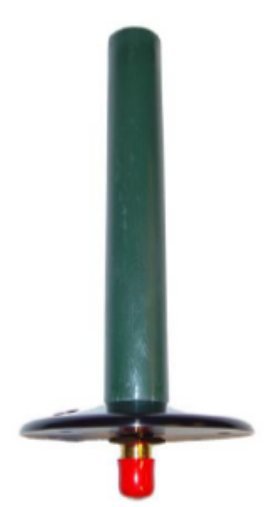

Fig. 27 - NAL Research SAF5350 Rod Antenna

\subsubsection{Iridium Short Burst Data Modem Circuit Card Assembly}

The Iridium SBD Modem CCA, shown in Fig. 25, consists of a COTS Iridium SBD data modem mounted on a custom PCB designed by the NRL.

The custom PCB provides PC/104 electrical and mechanical interfacing for the data modem, which is not in the PC/104 form factor. Electrical interfaces include power monitoring circuitry with a power good output signal, an Ethernet port with impedance matching circuitry, and a SBC interface.

The SBD data modem is shown in Fig. 26; specification highlights are listed below.

- Manufacturer: NAL Research Corporation

- Model: SBD9602E

- SBD only operation

- Configurable to DoD Enhanced Mobile Satellite Services (EMSS) Gateway

- 1616-1626.5 MHz operating frequency

- AT Command control

- Operating Temperature: $-40^{\circ} \mathrm{C}$ to $85^{\circ} \mathrm{C}$

- 1 x 15-Pin D-Sub connector

- 1 x SMA antenna connector 


\subsubsection{Iridium L-band Antenna}

The BPC Iridium L-band antenna is shown in Fig. 27; specification highlights are listed below.

- Manufacturer: NAL Research Corporation

- Model: SAF5350

- Quad-helix L-band operation

- 1610-1626.5 MHz frequency range

- High gain (+2 dB at zenith)

- Impact, UV, chemical and jet fuel resistant

\subsubsection{AC Power Adapter}

The BPC uses the same model AC power supply adapter as the RPC; it is described in Section 3.2.3.7.

\subsubsection{Wiring Diagram}

The BPC wiring diagram is shown in Fig. 28.

\section{SOFTWARE DESIGN}

\subsection{Host Platform Subsystem software module functionality}

The RPC and BPC use ARM processors running Linux. Once encrypted data packets are sent to the Iridium network they are received by the Iridium ground station server, and sent on to the ACRBS ground station.

- COM_19 - receives serial NMEA ASCII data from the GPS receiver, and converts the RMC sentences to Vehicle Tracking packets (ID 19). The ID 19 packets are then sent to DCC_SHIP.

- COM_40S - receives serial NMEA ASCII data from the AIS receiver in ITU-R M.1371-1 [2] format, and converts the NMEA data to ID 40 series packets. The ID 40 series packets are then sent to DCC_SHIP. Descriptions of each ID 40 series packet are contained in the Data Format Notes document [3].

- DCC_SHIP - assembles the ID 20 packet from the ID 19 and the ID 40 series packets. The ID 20 packet contains the position of the host platform and positions of AIS surface contacts within range of the host platform.

- ESP_RELAY - accepts packets from the encryption device (TACLANE) in the ESP-50 format IP packet it transmits. This module produces an ID 21 packet which encapsulates the ESP-50 packet, which contains the encrypted data to send to the Iridium network. It sends this ID 21 packet UDP to module VT_SBD. 


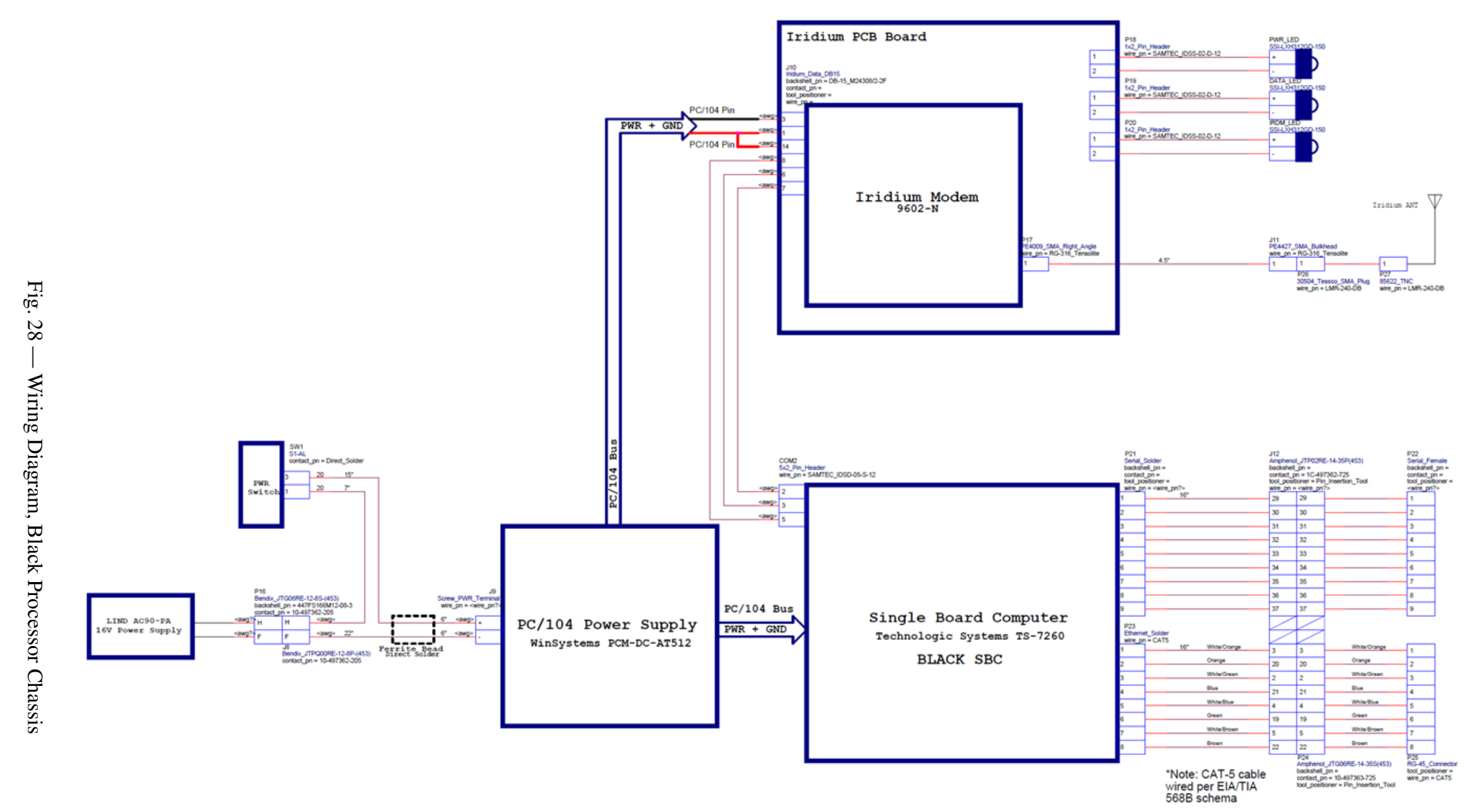


- VT_SBD - accepts packets to be sent to the Iridium modem. The module interacts with the modem, using SBD mode, and sends a packet when the modem is cleared to receive and send. This module buffers as necessary to accomodate dropouts and transmission errors, which occur normally, as the modem communicates with the satellite network. Note: max packet length is about 280 bytes

- CRBSPLAY - Test module for replaying stored position and AIS data. This is a utility to playback previously recorded NMEA data (.rcd files), and parcel the RMC and AIVDM sentences to the com_19 and com_40s port and IP addresses.

\subsubsection{COM_19 Module description}

The COM_19 module is described in this section (see Fig. 29 below).

- Overview

- Reads NMEA ASCII sentences via COM port or UDP port

- Processes NMEA RMC sentences, and outputs Vehicle Track (ID 19) packets to the specified UDP port

- Responds to function key F4 for status information

- Command line usage: com_19 P1 P2 P3 P4 P5

- P1 := Receive COM or UDP port

- P2 := Receive bit rate, or 0 if $\mathrm{P} 1$ is a UDP port

- P3 := Vehicle TX port (UDP Output port)

- P4 := Vehicle TX IP address (UDP IP address)

- P5 := Vehicle numeric ID

- Usage example: com_19 594404959 127.0.0.1 3009

- com_19 start up

- Reads command line and sets parameters in global memory

- Initializes the receiver for function key F4 processing. Function key F4 outputs status to screen using the printf function and returns control to main after processing F4

- Initializes the input ring buffer

- Starts Thread 1 and Thread 2 using the pthreads function

- Thread1 [com_in] description:

- Initializes the COM port or the UDP port

- Begins reading ASCII data and placing it onto the input ring buffer (ring_buf)

- When the ring buffer is full, increment the wrap counter (wrap), and set the push index (push) to zero

- Continue the operation until terminated 


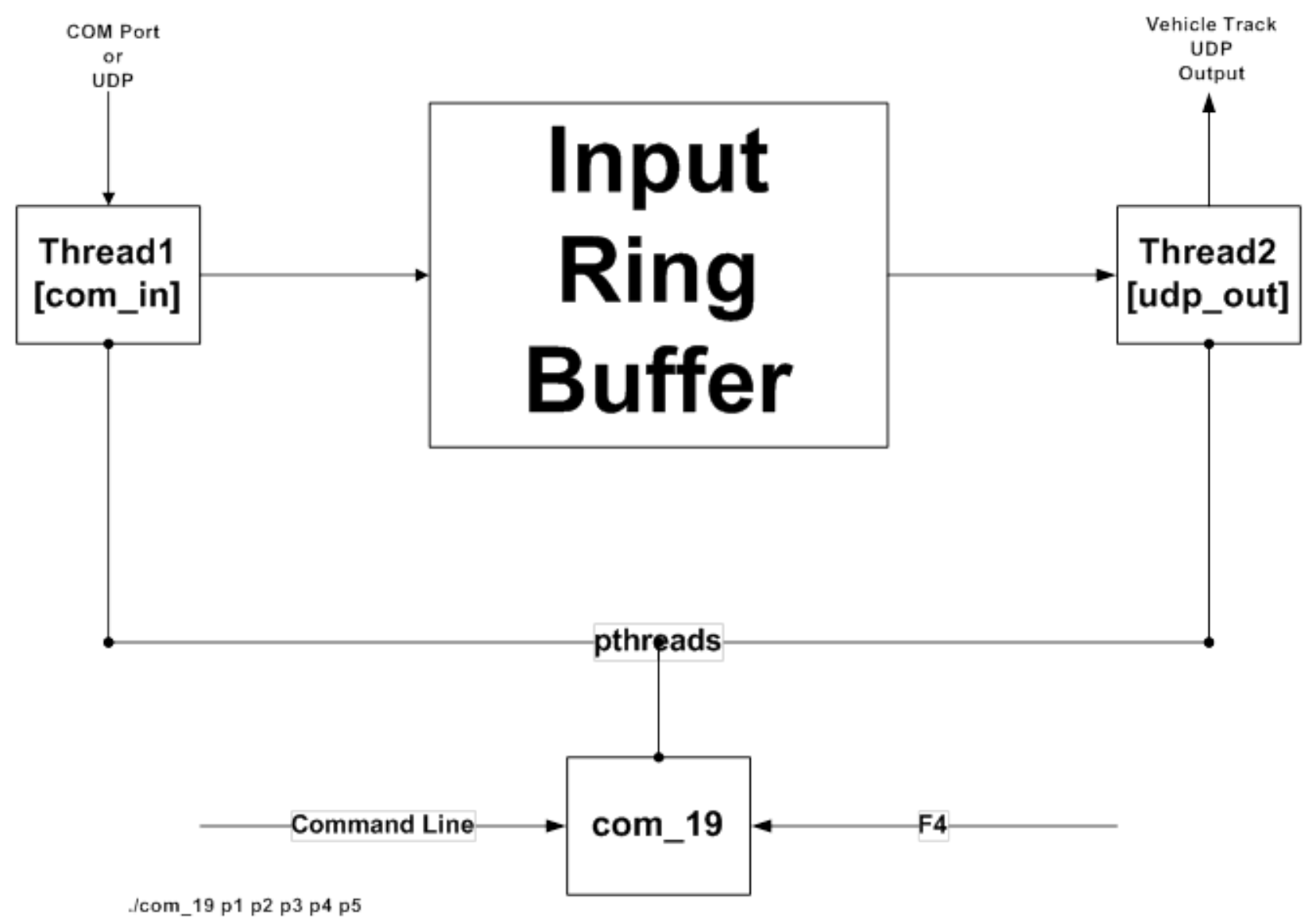

Fig. 29 - COM_19 Component Operations

- Thread2 [udp_out] description:

1. Set UDP output port

2. Loop a character at a time until an RMC sentence is found. Use normal ring buffer processing for data wraps.

3. Process RMC sentence and output a Vehicle track packet

4. Continue steps 2 and 3 until termination

- Known problems

1. None encountered

\subsubsection{COM_40S Module description}

The COM_40S module is described in this section (see Fig. 30 below).

- Overview 
- Reads NMEA ASCII sentences via COM port or UDP port

- Processes NMEA AIVDM sentences, and outputs AIS (ID 40 series) packets to the specified UDP port

- Responds to function key F4 for status information

- Command line usage: com_40s P1 P2 P3 P4 P5

- P1 := Receive COM or UDP port (Input communications port or UDP port)

- P2 := Receive bit rate, or 0 if $\mathrm{P} 1$ is a UDP port

- P3 := Vehicle TX port (UDP Output port)

- P4 := Vehicle TX IP address (UDP IP address)

- P5 := Vehicle numeric ID

- Usage example: com_40s 494404959 127.0.0.1 30009

- com_40s start up

- Reads command line and sets parameters in global memory

- Initializes the receiver for function key F4 processing. Function key F4 outputs status to screen using the printf function and returns control to main after processing F4

- Initializes the input ring buffer

- Starts Thread 1 and Thread 2 using the pthreads function

- Thread1 [com_in] description:

- Initializes the COM port or the UDP port

- Begins reading ASCII data and placing it onto the input ring buffere (ring_buf)

- When the ring buffer is full, increment the wrap counter (wrap), and set the push index (push) to zero

- Continue the operation until terminated

- Thread2 [udp_out] description:

1. Set UDP output port

2. Loop a character at a time until a AIVDM sentence is found. Use normal ring buffer processing for data wraps.

3. Process AIVDM sentences and outputs AIS (ID 40 series) packets

4. Continue steps 2 and 3 until termination

- Known problems

1. None encountered 


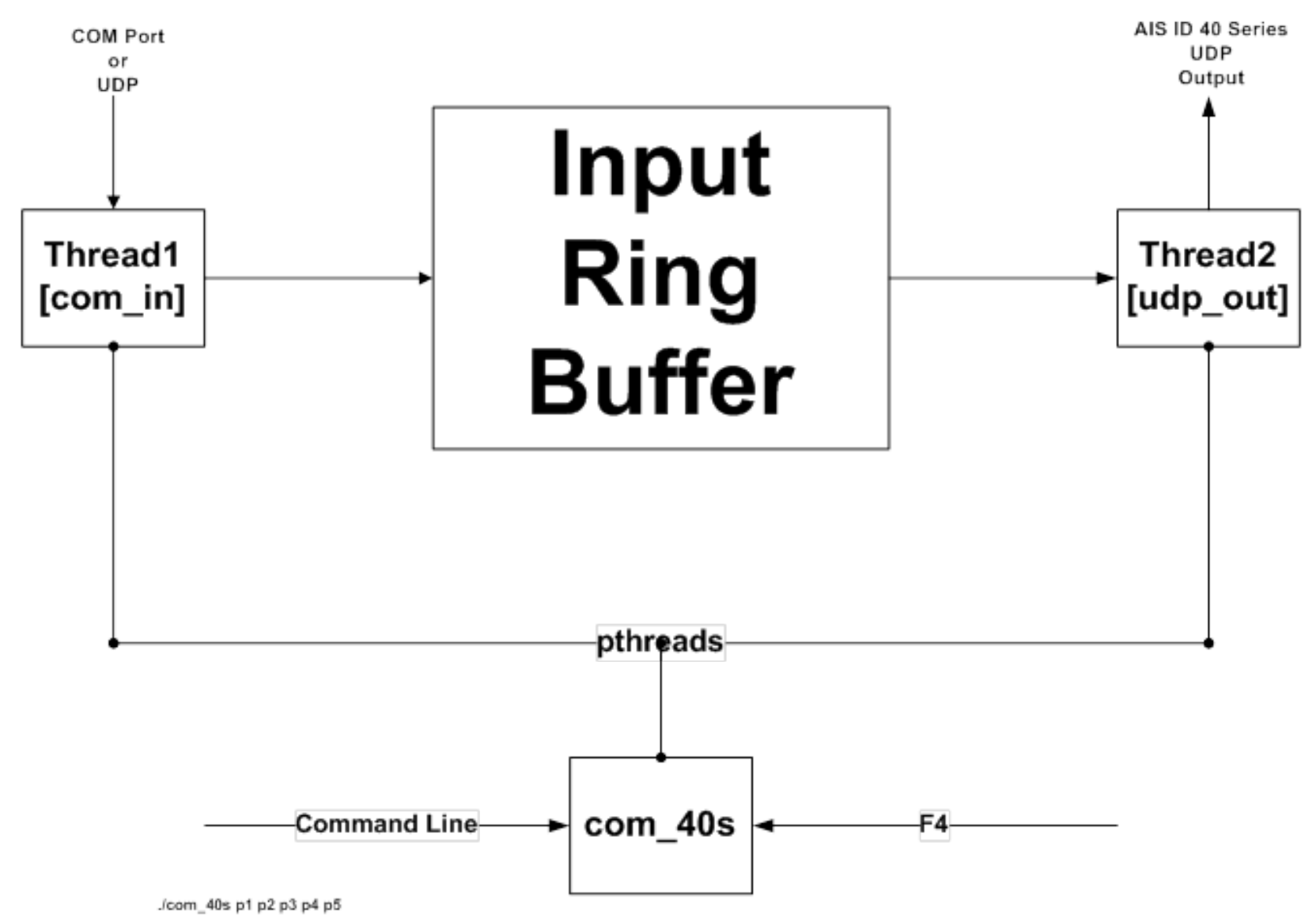

Fig. 30 - COM_40S Component Operations

\subsubsection{DCC_SHIP Module description}

The DCC_SHIP module is described in this section (see Fig. 31 and Fig. 32 below).

- Overview

- Reads Vehicle Track (ID 19) and AIS (ID 40 series) packets via UDP

- Processes packets and keeps a real-time track table

- Sends Ship Relay (ID 20) packets to another process which encrypts the packet or sends it to the shore processor

- Command line usage: dcc_ship P1 P2 P3 P4 P5 P6

- P1 := UDP Input port

- P2 := UDP Output port

- P3 := UDP Output IP Address 


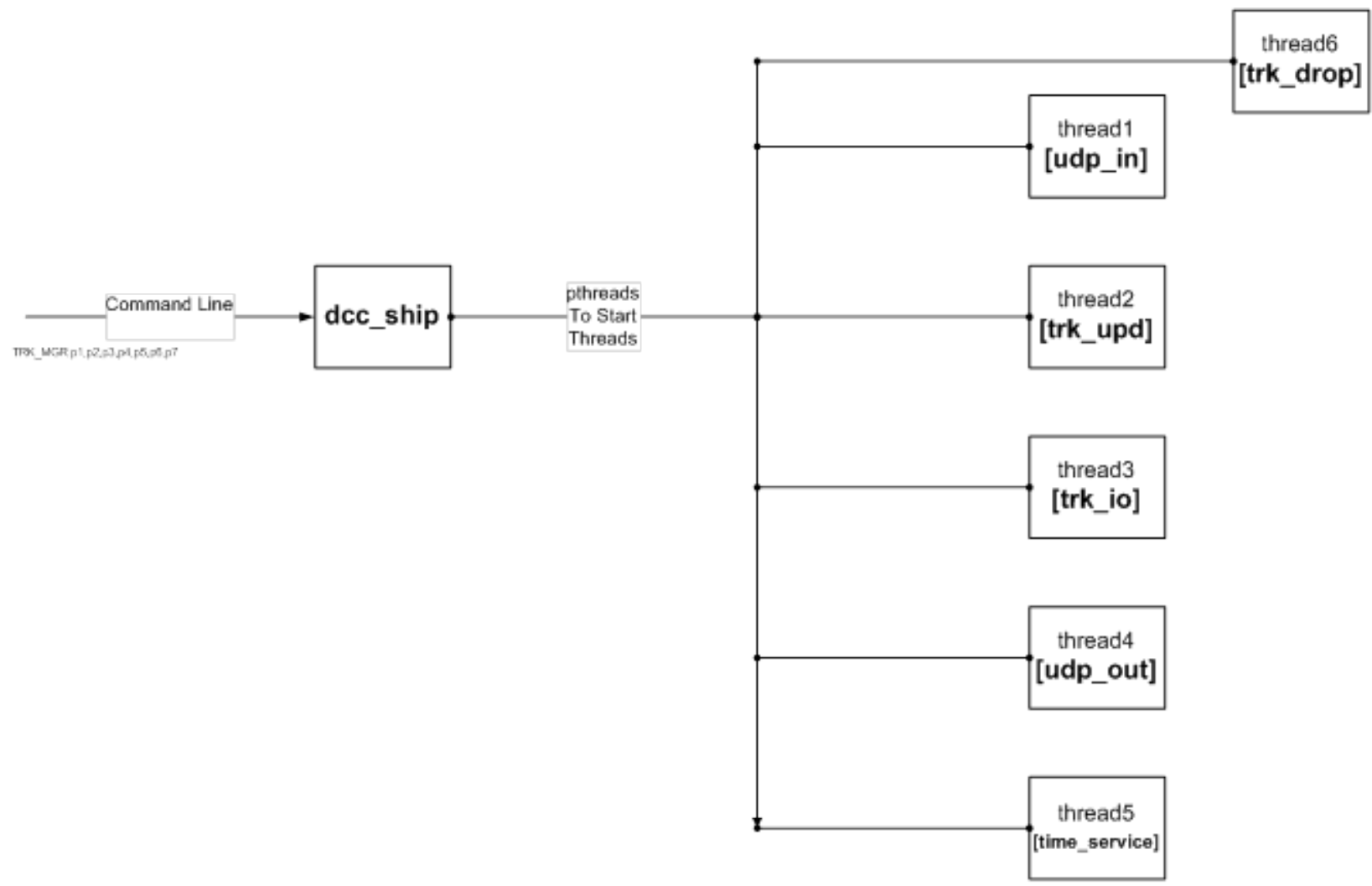

Fig. 31 - DCC_SHIP Threads 1 to 6

- P4 := track update interval in milliseconds

- P5 := timeout for dropping tracks

- P6 := platform ID

- Usage example: dcc_ship 49594971 127.0.0.1 2000090030009

- dcc_ship start up

- Reads command line and sets parameters in global memory

- Initializes the real-time track table

- Initialize ring buffers

- Starts threads 1 to 6 using the pthreads function

- Thread1 [udp_in] description:

- Initializes specified UDP input port

- Begins reading DFN packets and placing them onto the input ring buffer (ring_buf)

- When the ring buffer is full, increment the wrap counter (wrap), and set the push index (push) to zero 


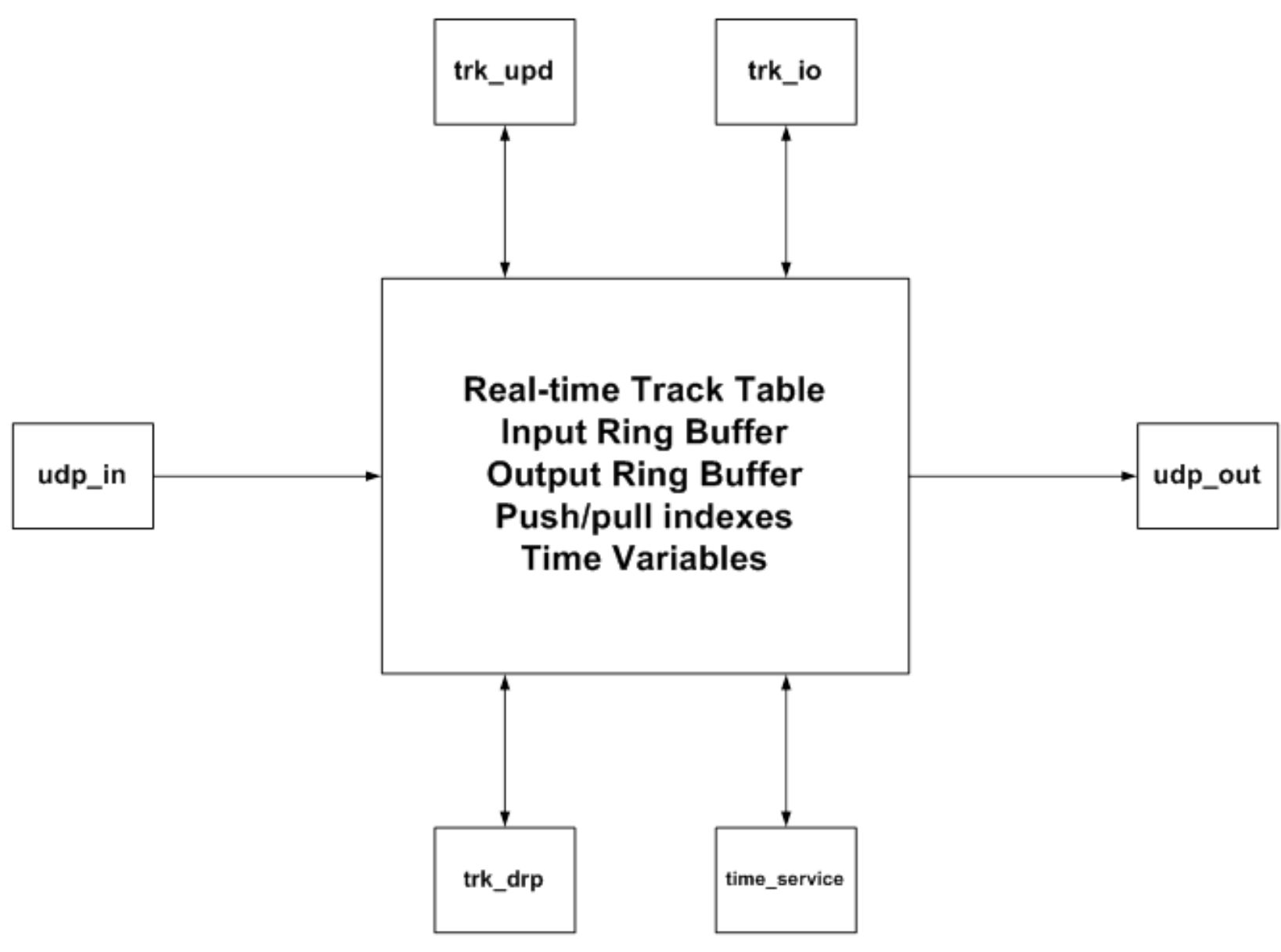

Fig. 32 - DCC_SHIP Component Operations

- Continue the operation until terminated

- Thread2 [trk_upd] description:

- Monitors the UDP input ring buffer for packets 19, 40, 41, 42, 43, 44, and 45

- Processes packet IDs 19, 40, 41, 42, 43, 44, and 45. Based upon the packet ID, a track is initiated if it is not active, or an active track is updated based upon the information contained in the packet

- The input ring buffer is monitored for a wrap, and the pull/wrap indicators are maintained

- Thread3 [trk_io] description:

- Process all tracks in the real-time track table to see if it is time to output the track. The output interval is based upon the track update interval (P4)

- If a track or tracks need to be output a ship relay packet is generated and placed upon the output ring buffer. If there are no tracks to output and and the specified update time has elapsed a Ship Replay packet with no tracks will be output 
- Thread4 [udp_out] description:

- Initializes UDP output port

- If a packet is on the output ring buffer it is sent to the output UDP port, and the pull index/wrap counter are updated if necessary

- Thread5 [time_service] description:

- The time service is run once per millisecond

- Using the gettimeofday system service the following are updated: j2000day, j2000sec, dostime

- Thread6 [trk_drop] description:

1. Search the real-time track table for a track that has not been updated for more than the P5 seconds

2. If a track meets this criteria the track number and drop track indicator are set to a value of -1 . This allows for the trk_io to delete the track.

- Known problems

1. Sometimes the metadata from one track gets set into another track. Other data remains intact. Eventually the condition clears itself. The condition is found only on the ARM processor. It is not repeatable on the Linux development system

\subsubsection{CRBSPLAY Module description}

- Overview

- Test module for replaying stored position and AIS data

- Command line usage: crbsplay P1,P2,P3,P4,P5,P6,P7,P8,P9,P10,P11,P12

- P1 := Input .rcd file

- P2 := ID21 VDM UDP Port \# 1

- P3 := ID21 VDM IP Address \# 1

- P4 := ID21 RMC UDP Port \# 2

- P5 := ID21 RMC IP Address \# 2

- P6 := RAW VDM UDP Port \# 3

- P7 := RAW VDM IP Address \# 3

- P8 := RAW RMC UDP Port \# 4

- P9 := RAW RMC IP Address \# 4

- P10 := Delay in milliseconds

- P11 := DEBUG MESSAGE SELECTION

- P12 := Platform ID

- Usage example: crbsplay pm050714.rcd,2944,127.0.0.1,2944,127.0.0.1,4944,10.250.14.8,5944,10.250.14.8,$1,0,30009$

- Known problems

1. None encountererd 


\subsection{Vehicle Tracking Subsystem software module functionality}

For integration and test, the ground processing is done in Building 240 of the NRL. An additional facility (Protasis Inc.) runs the packet distribution server. This server is the destination point of packets from the Iridium ground station, and is used to distribute UNCLASSIFIED packets that can be received and processed by the NRL. The servers are PCs running Linux. Processing at the NRL is done on one Linux-based desktop PC and two Windows-based laptops, a main laptop and a cart laptop.

The function of each software module is described below:

- VTLT - Accepts TCP/IP connections from the Iridium server site, which transmits packets received from the remote assets. This module removes the Iridium header and transmits the received packets to the local server module.

- VTL_SERV - Receives packets by UDP, and makes them available to one or more clients. Each client connects by TCP/IP and receives these packets. The ACRBS processing client connects from Building 240 of the NRL.

- SRV2UDP - This client module connects to the server at Protasis Inc. and receives the Iridium packets containing encrypted data. This module sends these packets via UDP to the module which decrypts them.

- ESP_SEND - Receives encrypted packets and sends them to the decryption device (TACLANE). Converts the packets to ESP50 format, which is expected by the TACLANE.

- VT_RPT - A "repeater" module, receiving a (UDP) stream of packets and sending them out one or more ports. The input packets are decrypted, so this module (and subsequent) is RED.

- DCC_CRBS - Accepts ASCII packets and differentiates between GPS and AIS packets, and transmits corresponding ID 25 and ID 40-series packets. This is only required for the prototype test configuration.

- TRK_MGR - This module is the Track Manager. It accepts packets which describe posits (position reports) and develops tracks - a moving representation of an asset with associated attributes (such as vehicle ID or name).

- VT_SERV - This is the Track Server module, which takes the (UDP) packet stream from TRK_MGR and sends it to connected clients. Currently, the only client is the GCCS display process, which connects via SRV2UDP.

- VT_MUX - This module accepts UDP packets and transmits them by serial link to the GCCS display system.

- VT_DEMUX - This module receives data from a serial link and re-forms packets into a UDP stream, for display by GCCS.

- DFN_MSG_FORMATTER - This program receives track packets (ID 60) and produces OTH-G formatted ASCII data packets.

- GCCS - This is the primary means of displaying data in the VTS. 


\section{VEHICLE TRACKING PACKET}

The VEHICLE TRACKING Packet is used to send the position and status of a vehicle to Vehicle Hub.

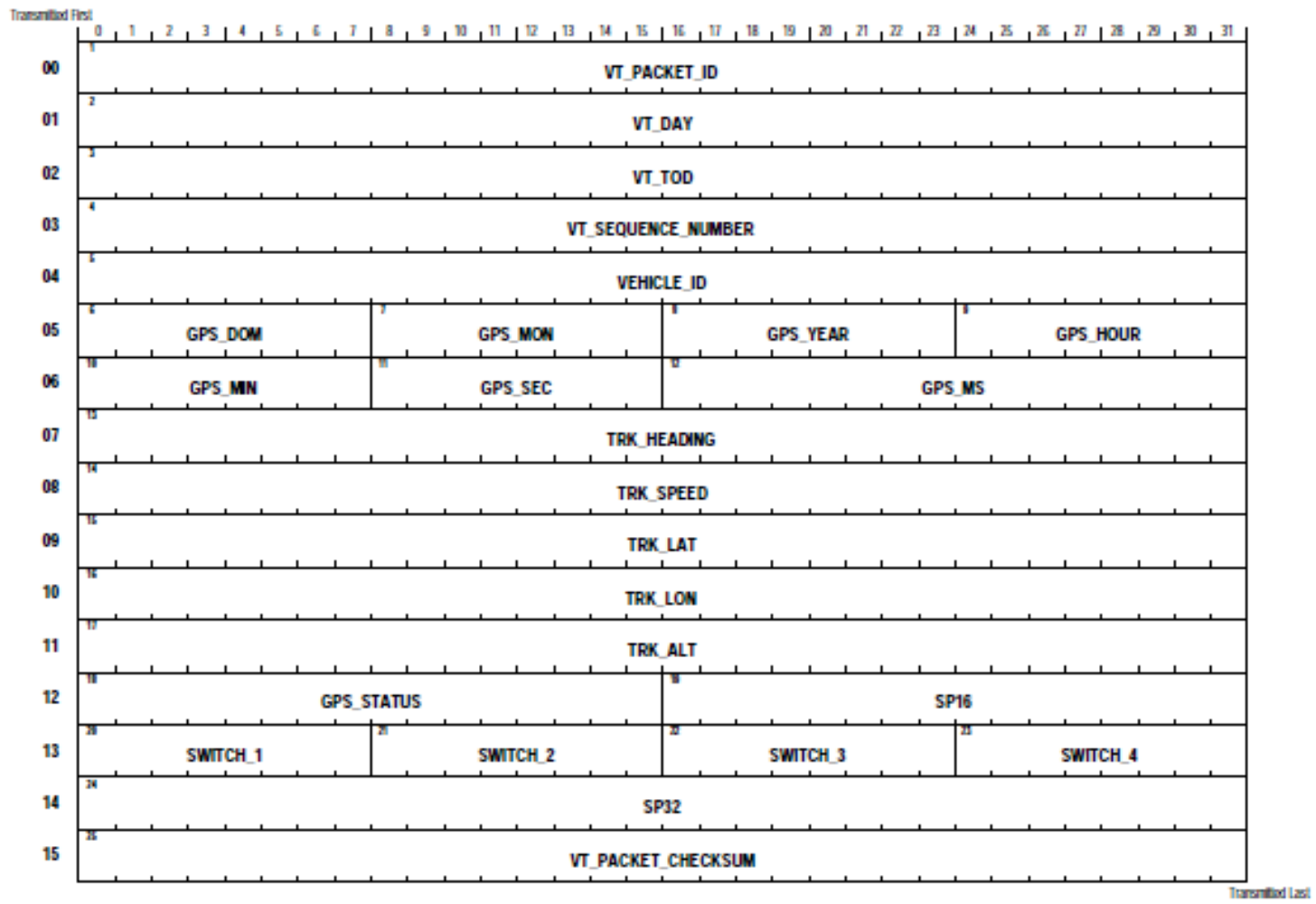

Fig. 33 - Data Packet Definition, ID 19 Vehicle Tracking Packet

- VTMSC - Test tool module which lists contents of asset reports in received (and decrypted) ID 20 packets.

\subsection{Data Packet Definitions}

The ID 19 data packet description is shown in Fig. 33. The ID 20 data packet description is shown in Fig. 34. The ID 21 data packet description is shown in Fig. 35. Descriptions of each field are contained in the Data Format Notes document [3].

\section{ENGINEERING TASKS COMPLETED}

\subsection{Host Platform Subsystem Hardware}

- Designed, fabricated, assembled, and tested first article prototypes of RPC and BPC using black anodized aluminum chassis parts 


\section{SHIP RELAY}

The SHIP Data Relay Packet provides a method for transmitting the relay platform position and relay observed target positions.

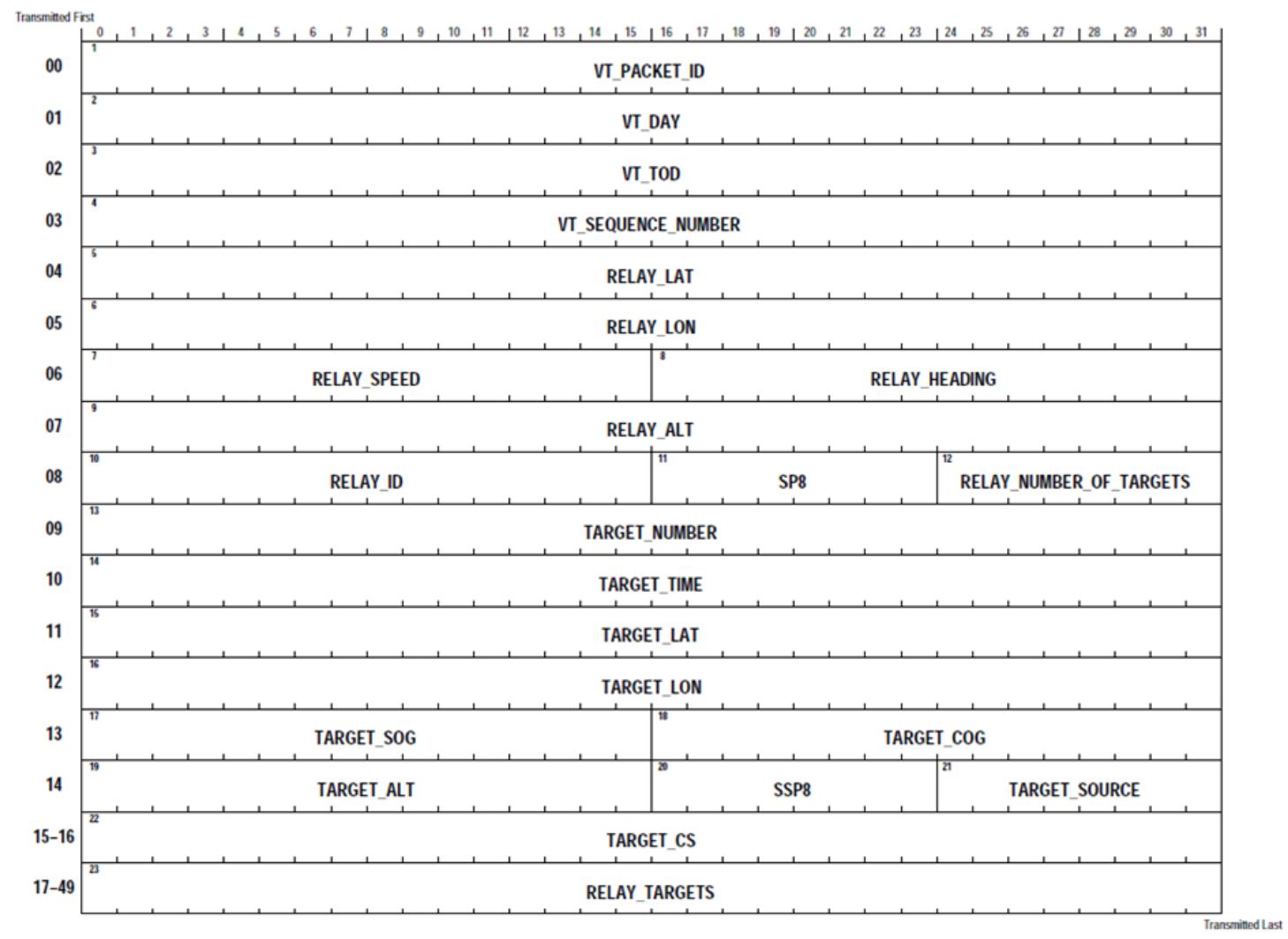

Fig. 34 - Data Packet Definition, ID 20 Ship Relay Packet 


\section{$00021 \quad$ ASCII RAW DATA PACKET}

The ASCII RAW DATA Packet is used to distribute raw ASCII data from various platforms. NOTE: This is a variable lenght packet. The length = VT_ASCII_RAW_DATA_PACKET_WORDS -127 + NUMBER_ASCII_BYTES/4.

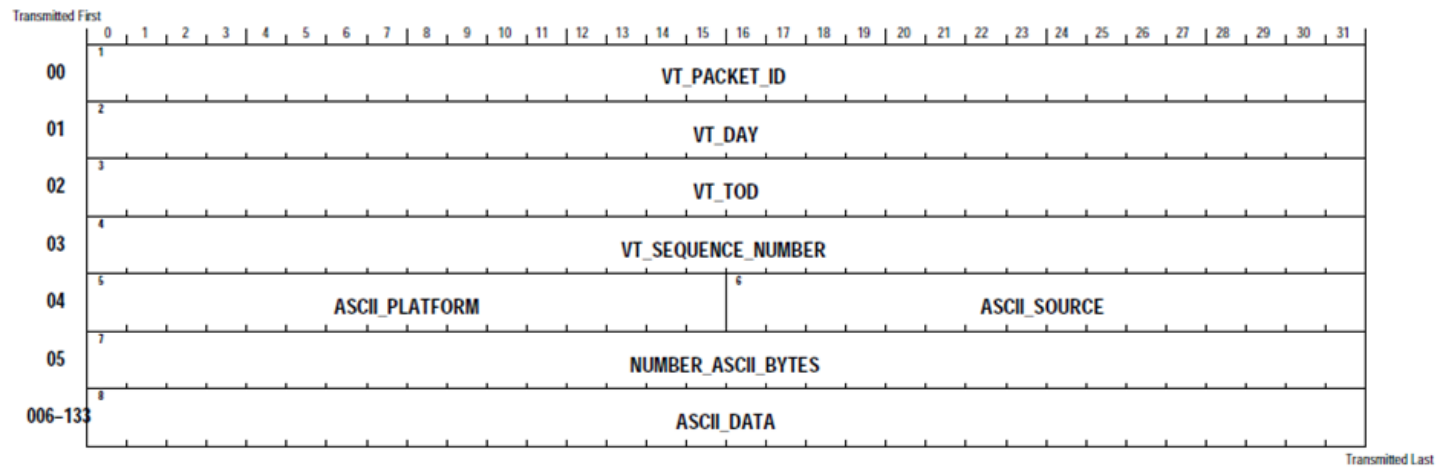

Fig. 35 - Data Packet Definition, ID 21 ASCII Raw Data Packet

- Designed, fabricated, assembled, and tested four prototype articles of RPC and BPC using nickelplated aluminum chassis parts for corrosion resistance

\subsection{Host Platform Subsystem Software}

- Designed and tested Class A AIS receiver interface

- Designed and tested GPS receiver interface

- Designed and tested Iridium 9602-N modem interface

- Designed and tested TACLANE-micro (KG-175D) interface

\subsection{Vehicle Tracking Subsystem Software}

- Designed and tested server application to receive SBD messages from the DoD Iridium Gateway client

- Designed and tested application to recover the encrypted payload from received messages

- Designed and tested data conversion to OTH-G message format (tested with GCCS-M)

\subsection{System Testing}

- Electromagnetic emissions and susceptibility testing of the HPS [4]. 
- Radio Frequency (RF) attenuation testing to determine maximum permissible antenna cable attenuation [5].

- An unclassified version of the HPS was tested on motor vessel My Turn over a span of more than one year in Florida waters without any hardware failures. Testing occurred while the vessel was moored pier side, and while the vessel was underway for numerous sea trials.

- Laboratory testing of Multiple Vessels-to-Shore testing via Iridium link. Specifically, four Host Platform Subsystems were installed in the NRL's Mobile Laboratory as shown in Fig. 36. Each HPS was connected to the Vessel Tracking Subsystem, which was housed in the NRL's Building 240. The AIS and GPS data was displayed on a GCCS-M station. Unclassified test encryption keys were used in all COMSEC devices. Data was monitored at each RPC, each BPC, the Black Server, and the Red Server. The testing ran continuously for several months.

\section{ENGINEERING TASKS REMAINING}

\subsection{Vehicle Tracking Subsystem Hardware}

- Build Linux-based Black Server, migrate software processes to the server, and test

- Build Windows-based Red Server, migrate software processes to the server, and test on unclassified network

- Migrate Red Server to classified network SIPRNet

- Obtain SIPRNet workstation accreditation for Red Server

- Coordinate data route between the NRLs SIPRNet and the MSCs SIPRNet

- Install PPK onto the NRL SIPRNet KG-175D and apply ACRBS configuration settings

- Stand-up GCCS-J system

\subsection{Deferred}

These tasks were identified as requirements creep and deferred to a future build

- Design and implementation of Data Classification toggle switch on the RPC front panel

- Develop the data path from the VTS to the HPS to allow for activities such as command, control, and pushing of remote updates.

\section{PROGRAM TASKS REMAINING}

\subsection{Candidate vessel selection}

- MSC 


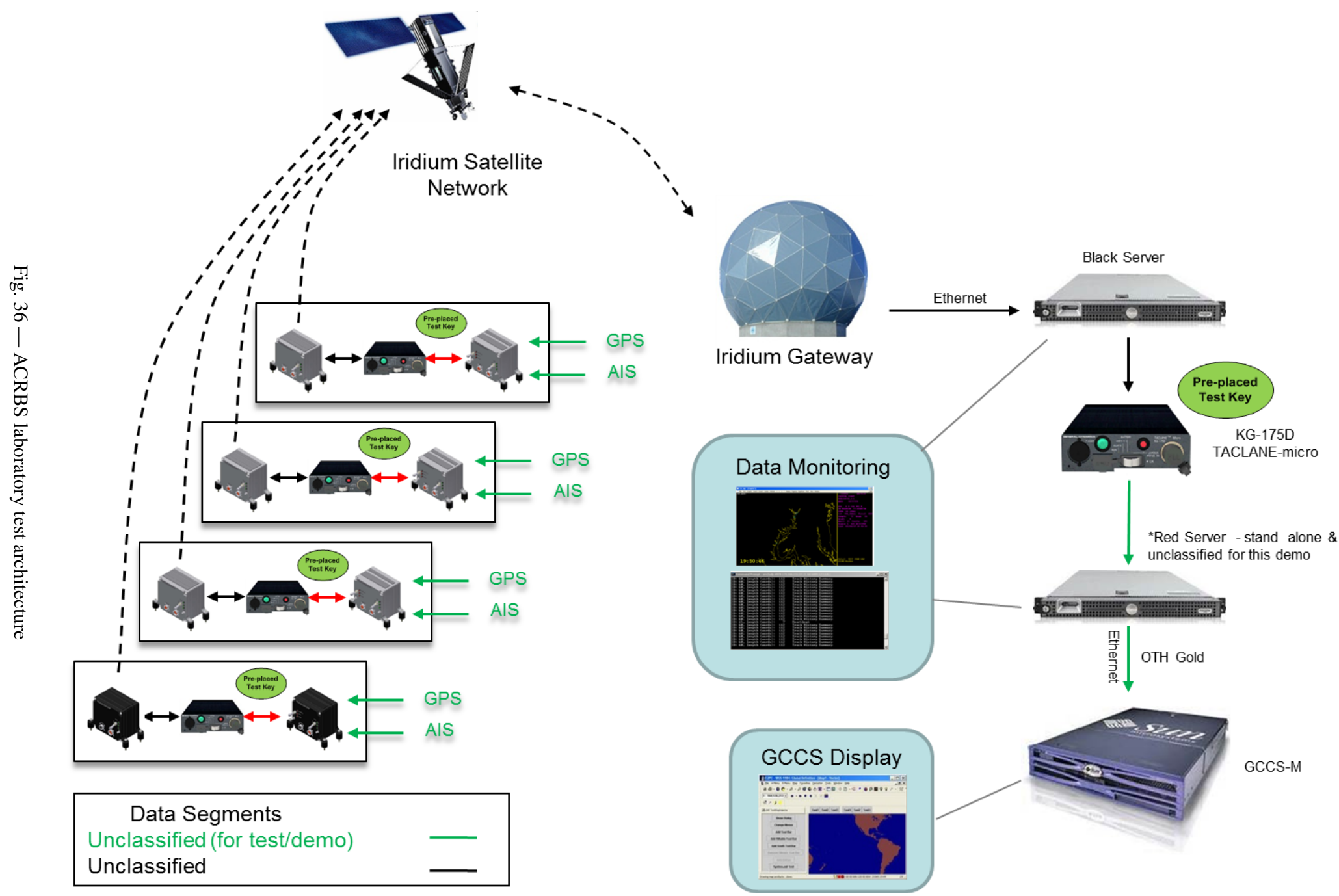


- Identify MSC vessels for prototype testing

- Determine if SHIPALT or TEMPALT is required for shipboard installation, develop the appropriate installation data package, and obtain approval for installation

- Determine component location and mounting requirements

- Determine cabling requirements (e.g. cable type, routing path, length)

\subsection{GCCS-J configuration}

- MSC

- Verify version number of GCCS-J at MSC

- NRL

- Obtain software load: NRL obtained GCCS-J v4.3

- Verify hardware configuration

- Install and test

\subsection{Documentation for Interim Authority to Test}

- NRL

- DIACAP Certification and Accreditation (C\&A) Plan for Type Accreditation

- DIACAP Plan of Action \& Milestones (POA\&M)

- Information Assurance and Operational Test Plans

\subsection{Host Platform Subsystem}

- NRL

- Evaluate ID 20 message format

\subsection{Vessel Tracking Subsystem}

- NRL

- Build one Windows-based and one Linux-based server

- Apply requirements of Security Technical Implementation Guide (STIG) to the operating systems and custom software applications

- Migrate software processes to servers and test 


\subsection{System tasks}

- MSC

- Obtain TACLANE devices for installations

- NRL

- Migrate VTS hardware/software to SIPRNet

- Create Crypto Key Management Plan

\section{7 $\quad$ Testing}

- NRL

- Verify OTH-G message delivery to GCCS-J; message delivery was verified to GCCS-M

- Document System Operational Parameters

\subsection{Transition}

- MSC

- Identify Program of Record

- MSC/NRL

- Transfer Iridium modem accounts to MSC

- Determine future design regarding crypto function (e.g embedded using Field Programmable Gate Array (FPGA))

- Establish funding profile to outfit MSC fleet

\section{SYSTEM OPERATIONS}

\subsection{Installation Considerations}

The ACRBS HPS is unobtrusive to the host vessel's architecture, only relying on the host for hardware mounting and electrical power. The following items are to be considered for a shipboard installation.

\subsubsection{Power}

The HPS requires three NEMA 5 outlets, one each for the RPC, BPC, and KG-175D TACLANE-micro COMSEC device. The HPS draws less than 40 Watts of electrical power. The appropriate regulations and procedures must be followed to ensure isolation of classified and unclassified equipment. 
Table 1 - Shipboard Component Location Considerations

\begin{tabular}{|l|l|}
\hline Component & Location Considerations \\
\hline Black Processor Chassis & $\begin{array}{l}\text { - Must be located in an internal space } \\
\text {-Should have easy access to the ship's exterior to accommodate } \\
\text { antenna cable runs }\end{array}$ \\
\hline Red Processor Chassis & $\begin{array}{l}\text {-Must be located in an internal space with the appropriate level } \\
\text { of physical security }\end{array}$ \\
\hline KG-175D TACLANE-micro & $\begin{array}{l}\text { - Must be located in an internal space with the appropriate level } \\
\text { of physical security }\end{array}$ \\
\hline Antennas, general & $\begin{array}{l}\text {-Should not be located near high powered ( }>15 \mathrm{~W} \text { AEP) emitters } \\
\text { (e.g. RADAR) with operating frequencies between } 150 \mathrm{MHz} \text { and } \\
\text { 160 MHz or between } 1.5 \mathrm{GHz} \text { and } 1.8 \mathrm{GHz}\end{array}$ \\
\hline GPS and Iridium antennas & $\begin{array}{l}\text {-Should have a clear view of the sky ( }>15 \text { degrees elevation, 360 } \\
\text { degrees in azimuth) }\end{array}$ \\
\hline AIS antenna & -Should have a clear view of as much of the horizon as possible \\
\hline
\end{tabular}

\subsubsection{Component Locations}

In general, the RPC, BPC, and TACLANE-micro should be located near one other for ease of cabling; however, appropriate regulations and procedures must be followed to ensure isolation of classified and unclassified equipment. Refer to Table 1 for considerations when choosing component locations.

\subsubsection{Antenna Mounting}

Antennas should be mounted with a clear view of the sky to prevent obstruction of the system's receivers and transmitter, a sufficient distance from high power emitters (e.g. RADAR) to avoid interference, and without excessive cable run lengths to preserve signal integrity. Table 2 lists the maximum permitted cable run of the Iridium antenna for several cable types based on $10 \mathrm{~dB}$ of loss. These results were determined from RF attenuation testing performed at the NRL [5]. These guidelines may also be followed for the AIS and GPS antenna cables.

\subsubsection{Communications Security Device Mounting Tray}

The tray Fig. 37 in may be considered for shipboard installation of the KG-175D device; otherwise, a custom tray may be designed. Specification highlights of the tray are listed below. Note that the HPS uses only one COMSEC device.

- Manufacturer: General Dynamics

- Model: MC-106A

- Mounting space for three TACLANE devices

- Rear cooling fins for thermal stability 
Table 2 - Maximum Length of Iridium Antenna Cable Run

\begin{tabular}{|l|c|c|}
\hline RF Cable Type & Loss per $100 \mathrm{ft}(\mathrm{dB})$ & ${\text { Maximum Run }(\mathrm{ft})^{\mathrm{a}}}^{\mathrm{a}}$ \\
\hline LMR-195 & 15.118 & 66.15 \\
\hline LMR-200 & 13.445 & 74.38 \\
\hline LMR-240 & 10.273 & 97.34 \\
\hline LMR-400 & 5.341 & 187.23 \\
\hline RG-58 & 19.903 & 50.24 \\
\hline RF-59 & 14.914 & 67.05 \\
\hline
\end{tabular}

a Maximum run is based on $10 \mathrm{~dB}$ of total signal loss

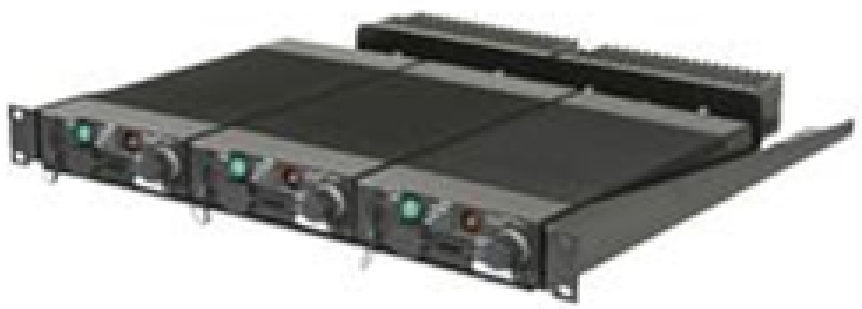

Fig. 37 - TACLANE KG-175D Mounting Tray

\section{RECOMMENDATIONS}

1. Replace KG-175D with C100 TACLANE; reference Section 9.1

2. Combine the RPC and BPC into one unit with embedded COMSEC; reference Section 9.2

3. Integrate additional sensing systems to help improve situational awareness; reference Section 9.3

\subsection{Communications Security Device, TACLANE-C100}

This recommendation is to replace the KG-175D with the TACLANE-C100 to enable a common implementation on host platforms regardless of the platform's ability to possess CCI. The C100, shown in Fig. 38, is not a CCI, and it has the same physical form factor as the KG-175D. Manufacturer's listed features include:

- Manufacturer: General Dynamics 


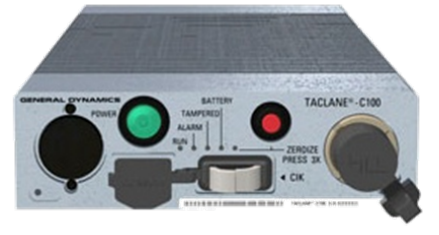

(a)

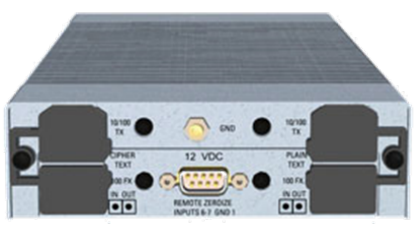

(b)

Fig. 38 - TACLANE KG-175D. (a) Front. (b) Rear.

- Model: C100

- HAIPE v4.1 Certified

- NSA Certified to Protect Information Classified Secret and Below

- Foreign Interoperability and Suite B Compatible

- Simplified Non-CCI Handling Supports "Leave Behind Deployment"

\subsection{Single unit Host Platform Subsystem}

This recommendation is to replace the current HPS consisting of the RPC, BPC, and TACLANE KG$175 \mathrm{D}$ with a single unit containing a programmable embedded crypto module and the supporting RED and BLACK data processing functions. Issues to consider are listed below:

1. Improved flexiblity and maintainability

- COMSEC requirements have tended to evolve over time. There is a risk that the current ACRBS would require modification in all three HPS components (RPC, BPC, and crypto) to meet future COMSEC requirements (e.g. for new algorithms/crypto keys). Combining RED/BLACK frontend processing with embedded crypto into a custom-built single unit would simplify the process of designing hardware/software to accomodate anticipated future COMSEC requirements.

\section{Reduced hardware costs}

- The hardware costs associated with a single unit are likely to be less than for a HPS. Only one enclosure would be needed, and components that the RPC and BPC have in common (e.g. SBC, PSM) could be potentially shared provided that the RED and BLACK processing streams are kept separate. An embedded crypto module is likely to cost less than a TACLANE KG-175D.

3. Improved physical security

- A single-unit design would simplify physical security to the need to account only for a single unit and its interfaces. 


\subsection{Integrate additional sensing systems into ACRBS}

This recommendation is for integration of additional non-AIS collection systems and associated hardware/software into ACRBS to help improve situational awareness (e.g. RADAR, Automatic dependent surveillance-broadcast (ADS-B)) . The ACRBS architecture will readily accept sensor data in any packetized form. For example, the NRL has demonstrated that the Track Manager (TRK_MGR) software module can ingest packetized ship RADAR feeds.

\section{REFERENCES}

1. NMEA 0183 Standard For Interfacing Marine Electronic Devices (NMEA, June 2012), Version 4.10.

2. RECOMMENDATION ITU-R M.1371-1, Technical characteristics for a universal shipborne automatic identification system using time division multiple access in the VHF maritime mobile band (ITU, 19982001).

3. W. Mall, "Data Format Notes," Version 133 (February 2014).

4. E. Vandersall, "Automated Identification System Collection and Reachback System (ACRBS) Electromagnetic Compatibility Test Report," rept., Naval Research Laboratory (March 2013).

5. E. Vandersall, "Automated Identification System Collection and Reachback System (ACRBS) Radio Frequency Attenuation Test,” rept., Naval Research Laboratory (November 2013).

\section{ACKNOWLEDGMENTS}

The authors recognize Tracy L. Hill, Protasis Inc., for his role in the development of ACRBS software. 



\section{Appendix A}

\section{LIST OF ACRONYMS}

AIS Automatic Identification System

AIVDM AIS position reports from other vessels

ACRBS AIS Collection and Reach-back System

ADS-B Automatic dependent surveillance-broadcast

BPC Black Processor Chassis

C\&A Certification and Accreditation

CCA Circuit Card Assembly

CCI Controlled Cryptographic Item

COM Communications

COMSEC Communications Security

COP Common Operational Picture

COTS Commercial Off-The-Shelf

DFN Data Format Notes

DIACAP DoD Information Assurance Certification and Accreditation Process

DISN Defense Information System Network

DoD Department of Defense

EMSS Enhanced Mobile Satellite Services

ESP Encapsulated Security Protocol

FPGA Field Programmable Gate Array

GCCS Global Command and Control System

GCCS-J Global Command \& Control System - Joint

GCCS-M Global Command \& Control System - Maritime

GPS Global Positioning System

HPS Host Platform Subsystem 
IATT Interim Authority to Test

IP Internet Protocol

MSC Military Sealift Command

NEMA National Electrical Manufacturers Association

NMEA National Marine Electronics Association

NRL Naval Research Laboratory

OTH-G Over-the-Horizon-GOLD

OV-1 Operational Viewpoint

PCB Printed Circuit Board

POA\&M Plan of Action \& Milestones

PPK Pre-placed key

PSM Power Supply Module

RF Radio Frequency

RMC Recommended Minimum Sentence-C

RPC Red Processor Chassis

RRF Ready Reserve Force

SBC Single Board Computer

SBD Short Burst Data

SIPRNet Secret Internet Protocol Router Network

SMA Sub-miniature version A

STIG Security Technical Implementation Guide

SvcV-1 Services Viewpoint

TACLANE Tactical Local Area Network Encryption

TCP/IP Transmission Control Protocol/Internet Protocol

UDP User Datagram Protocol

USTRANSCOM U.S. Transportation Command

VHF Very High Frequency

VTS Vehicle Tracking Subsystem 